GA-A- -20803

D.92 009646

\title{
FUSION PROGRAMS IN APPLIED PLASMA PHYSICS
}

FISCAL YEARS 1989 THROUGH 1991

FINAL REPORT

by

PROJECT STAFF

\author{
Work prepared under \\ Department of Energy \\ Contract DE-AC03-89ER53277
}

GENERAL ATOMICS PROJECT 3468

DATE PUBLISHED: FEBRUARY 1992

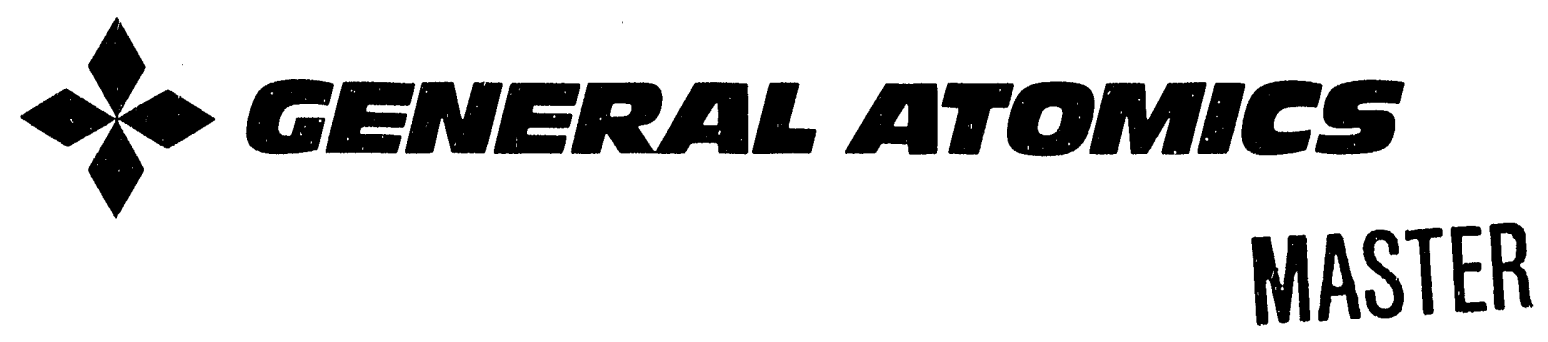




\section{DISCLAIMER}

This report was prepared as an account of work sponsored by an agency of the United States Government. Neither the United States Government nor any agency thereof, nor any of their employees, makes any warranty, express or implied, or assumes any legal liability or responsibility for the accuracy, completeness, or usefulness of any information, apparatus, product, or process disclosed, or represents that its use would not infringe privately owned rights. Reference herein to any specific comrrercial product, process, or service by trade name, trademark, manufacturer, or otherwise, does not necessarily constitute or imply its endorsement, recommendation, or favoring by the United States Government or any agency thereof. The views and opinions of authors expressed herein do not necessarily state or reflect those of the United States Government or any agency thereof.

This report has been reproduced directly from the best available copy

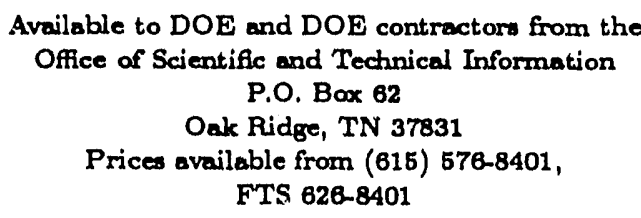

Available to the public from the

National Technical Information Service

U.S. Department of Commerce 5285 Port Royal Road Springfield, VA 22161 


\section{CONTENTS}

1. APPLIED PLASMA PHYSICS OVERVIEW $\ldots \ldots \ldots \ldots \ldots \ldots \ldots$. $\ldots \ldots$

2. THEORETICAL SCIENCE PROGRAM $\ldots \ldots \ldots \ldots \ldots$. . . . . .

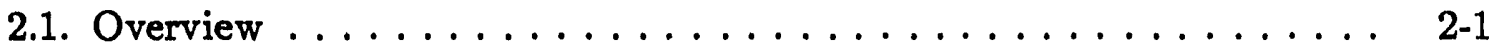

2.1.1. FY89-FY91 APP Theory $\ldots \ldots \ldots \ldots \ldots \ldots \ldots \ldots \ldots$ 2-3

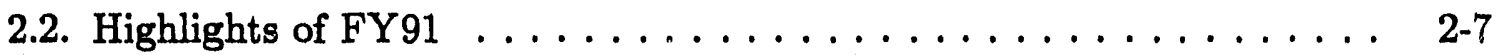

2.2.1. Numerical Study of a Singular Differential Equation

Relevant for the Finite $\beta$ Tearing Mode in a

Toroidal Plasma (GA-A20135) . . . . . . . . . . . . . . 2-9

2.2.2. Isotopic Scaling in the Drift Wave Model (GA-A20182) . . . 2 2-15

2.2.3. Electromagnetic Effects in Fluctuations of

Non-Equilibrium Plasmas and Application to

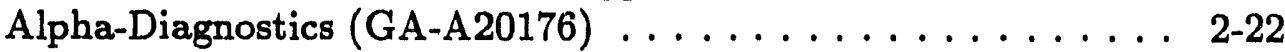

2.2.4. FY91 Publications $\ldots \ldots \ldots \ldots \ldots \ldots \ldots \ldots \ldots \ldots$ 2-27

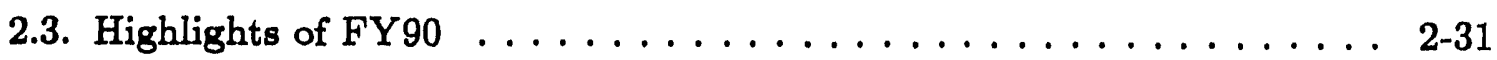

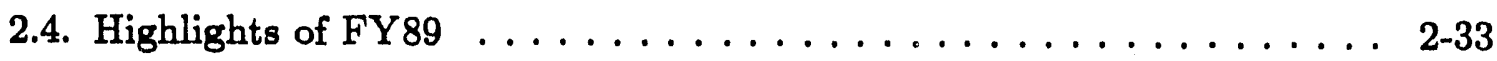

2.5. References for Section $2 \ldots \ldots \ldots \ldots \ldots \ldots \ldots \ldots \ldots \ldots$ 2-34

3. ALPHA PARTICLE DIAGNOSTIC $\ldots \ldots \ldots \ldots \ldots \ldots \ldots \ldots$ 3-1

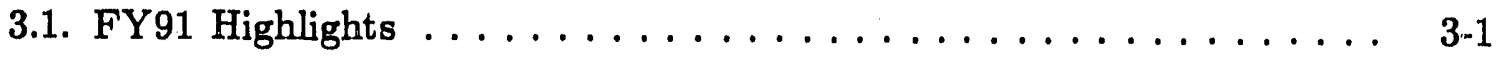

3.1.1. Use of Lithium Pellet Clond Target . . . . . . . . . . . . . 3-1

3.1.2. Fall 1991 Neutral Measurements on TFTR . . . . . . . . . 3-5

3.1.3. 1992 TFTR Experiments $\ldots \ldots \ldots \ldots \ldots \ldots \ldots \ldots \ldots$. $\ldots$. 6

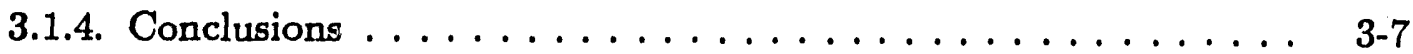

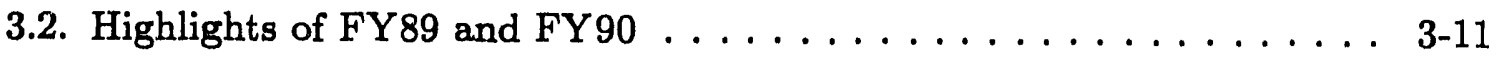

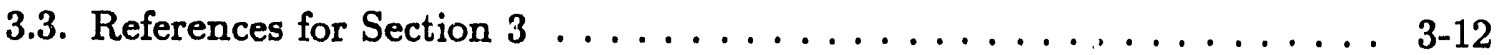

4. CURRENT DENSITY DIAGNOSTIC $\ldots \ldots \ldots \ldots \ldots \ldots$ 4-1

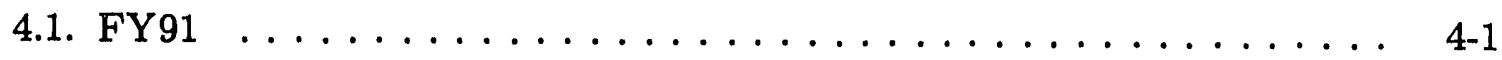

4.1.1. Li Beam Modifications . . . . . . . . . . . . . 4-1

4.1.2. Fluorescence Detection System . . . . . . . . . . . 4-1

4.1.3. Data Analysis $\ldots \ldots \ldots \ldots \ldots \ldots \ldots \ldots \ldots \ldots$ 4-3 


\section{CONTENTS (Continued)}

4.2. FY89-90 Highlights: Current Density Diagnostic $\ldots \ldots \ldots \ldots$. . . 4-6

4,2.1. Laser Enhanced Attenuation $\ldots \ldots \ldots \ldots \ldots \ldots \ldots$. . . . . . .

4.2.2. Zeeman Polarimetry . . . . . . . . . . . . . . 4-7

4.2.3. Edge Density Diagnostic Using Low Energy Li Beam . . . . . . 4-7

4.3. References for Section $4 \ldots \ldots \ldots \ldots \ldots$. . . . . . . . . . . . . .

5. FUSION USER SERVICE CENTER $\ldots \ldots \ldots \ldots \ldots \ldots \ldots \ldots \ldots$ 5-1

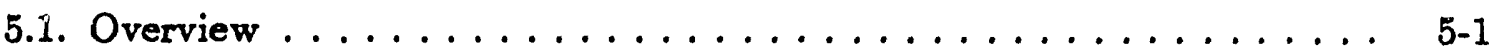

5.2. FY91 Major Accomplishments $\ldots \ldots \ldots \ldots \ldots \ldots \ldots \ldots \ldots$ 5-1

\section{FIGURES}

3-1. $\mathrm{Li}^{+}$light at $5485 \AA$ from a lithium pellet injected into TFTR $\ldots \ldots$ 3-2

3-2. Calculated neutral equilibrium fractions for ${ }^{4} \mathrm{He}$ passing through a $\mathrm{Li}^{+}$target and a $\mathrm{C}^{4+}$ target $\ldots \ldots \ldots \ldots \ldots \ldots \ldots \ldots$

3-3. Fall 1991 neutral particle analyze:: designed to observe ${ }^{3} \mathrm{He}$ and D neutrals from TFTR .................

3-4. Schematic of high energy analyzer needed for measuring $37 \mathrm{MeV}$ alphas from $\mathrm{D}-{ }^{3} \mathrm{He}$ reactions in 1992 and $3.5 \mathrm{MeV}$ alphas from $\mathrm{D}-\mathrm{T}$ reactions in $1993-94 \ldots \ldots \ldots \ldots \ldots$

3-5. Calculated ${ }^{3} \mathrm{He}$ signal levels fron a modified TFTR neutral

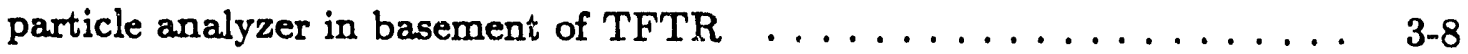

3-6. Calculated $3.7 \mathrm{MeV}{ }^{4} \mathrm{He}$ signal from alphas created by ${ }^{3} \mathrm{He}-\mathrm{D}$ reactions in ICH heated TFTR plasmas . . . . . . . . . . . 3-9

3-7. Calculated 3.5 $\mathrm{MeV}^{4} \mathrm{He}$ signal during TFTR D-T experiment

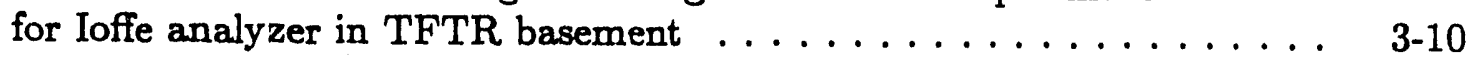

4-1 Schematic of the $\mathrm{Li}$ beam configured for operation at energies

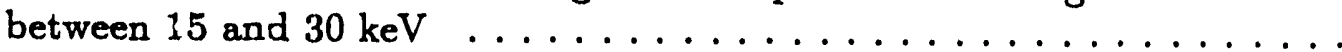

4-2. A model edge plasma density profile including fluctuations and the Li beam fluorescence profile predicted by the nine-state code . . . . 4-4

4-3. The edge density profile derived from the fluorescence profile in Fig. 4-2 using the two-state algorithm and the original edge

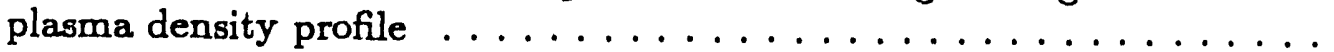

4-4. Schematic of the transmitted beam detector with amplification

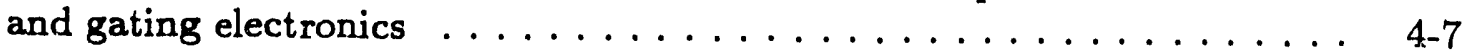

4-5. (a) Measured pitch angle and (b) TEXT plasma current and density . . . 4-8

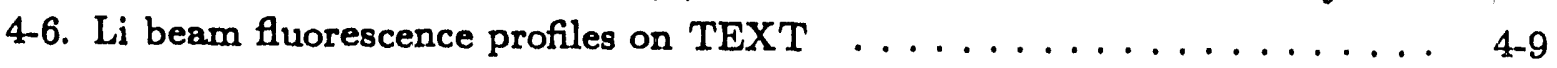




\section{APPLIED PLASMA PHYSICS OVERVIEW}

Generd Atomics' (GA) effort in Applied Plasma Physics consists of a theoretical and an experimental program. The theoretical program comprises of an interconnected series of investigations designed to elucidate the equilibrium, stabililty, and confinement properties of magnetically confied plasmas in general; shaped high $\beta$ tokamak plasmas in particular. An important component of the program is our research in the application of $\mathrm{rf}$ waves and particle beams to enhance the performance of tokamaks. The objectives of the theoretical science program are:

1. To support the interpretation of present experirients and predict the outcome of future planned experiments.

2. To improve on existing models and codes and validate against experimental results.

3. To conduct theoretical physics development of advanced concepts with applications for DIII-D and future devices.

Major accomplishments in FY91 include the corroboration between theory and experiment on MHD behavior in the second stable regime of operation on DIII-D, and the frequency and mode structure of toroidal Alfvén eigenmoces (TAEs) in high beta, shaped plasmas. We have made significant advances in the development of the gyro-Landau fluid approach to turbulence simulation which more accurately models kinetic drive and damping mechanisms. Several theoretical models to explain the bifurcation phemomenon in $\mathrm{L}-$ to $\mathrm{H}$-mode transition were proposed providing the theoretical basis for future experimental verification. The capabilities of new rf codes have been upgraded in response to the expanding needs of the rf experiments. Codes are being employed to plan for a fully non-inductive current drive experiment in a high beta, enhanced confinement regime.

GA's experimental effort in Applied Plasma Physics encompasses two advanced diagnostics essential for the operation of future fusion experiments:

1. Alpha particle diagnostic

2. Current and density profile diagnostics 
The diagnostic development program continued to make significant contributions to two difficult measurements needed by the tokamak community: alpha particle distribution and confinement measurements, and current density profile measurements. In FY91, a neutral particle analyzer was developed and installed on TFTR to be ready for a first test of the alpha diagnostic using lithium pellet during $\mathrm{D}-{ }^{3} \mathrm{He}$ ICRH and deuterium NBI experiments in FY92. The lithium beam diagnostics on TEXT have been ugraded and reinstalled but the experiment was delayed until FY92 due to coil problems.

The majority of the GA fusion staff makes use of the computers at the GA USC (User Service Center), either directly or as a node connected to the National Energy Research Supercomputer Center (NERSC) network. The availability of the system was again of very high quality in the past year.

Major accomplishments in FY90 included the corroboration between theory and experiment on MHD behavior in high $\beta$ plasmas, which expanded the boundary of tokamak operations; elucidation of turbulence based confinement characteristics in $\mathrm{H}$-mode, which might confirm the feasibility of a "wind tunnel" experiment for projection to ignition; development of a self-consistent neoclassical transport theory for the plasma edge, winich provided a better understanding of $\mathrm{L}-$ to $\mathrm{H}$-mode transition in divertor tokamaks; nnd detailed modeling, which resulted in enhanced understanding, of $\mathrm{ff}$ heating experiments. Novel applications of of for current drive (with the goal of developing high efficiency current drive for ITER) and plasma diagnostics have also been explored. In experimental Applied Plasma Physics, we have been able to corroborate the measured penetration distance of carbon pellet with a theoretical model. Furthermore, the observed size of the carbon cloud substantiated the applicability of this technique for a D-T experiment.

Major accomplishments in FY89 included detailed comparisons between theory and experiment on MHD behavior in high $\beta$ plasmas; elucidation of $\eta_{\mathrm{i}}$ mode and drift wave characteristics in $\mathrm{H}$-mode, development of a self-consistent neoclassical transport theory for the plasma edge; and detailed modeling, which resulted in enhanced understanding, of rf heating experiments. The diagnostic development program continued to make advances in alpha particle diagnostics and current density profile measurements. The use of resonant helical perturbations was used to study the effects and applications of an ergodic magnetic limiter and a resonant helical divertor. A key accomplishment of the USC was the installation of a mini-supercomputer to handle the rapidly increasing demand of the DIII-D data analysis task. 


\section{THEORETICAL SCIENCE PROGRAM}

\subsection{OVERVIEW}

The objective of the theoretical plasma physics research at GA is to support the DIII-D and other tokamak experiments and to significantly advance our ability to design a commercially-attractive fusion reactor. We categorize our efforts in three areas: magnetohydrodynamic (MHD) equilibria and stability, plasma transport with emphasis on $\mathrm{H}$-mode, divertor and boundary physics, and radio frequency (rf). The following list provides a brief review of our recent accomplishments.

In the MHD area, we have accomplished the following:

- Developed techniques for achieving reactor-relevant $\beta$ and high $I_{\mathrm{p}}$.

- Ascertained the effects of elongation and shaping on stability thereby identifying stable operating windows in safety factor and current.

- Achieved new and important understanding of the relationship between plasma configuration and the current and pressure profiles in divertor discharges, accounting for bootstrap currents and alpha particle pressure.

The close interaction between theory and experiment in the MHD area has again resulted in major advances. An extensive mapping of the $\beta$ limit as a function of the plasma profiles has been carried out using our numerical equilibrium and stability codes. Guided by this, DIII-D was able to achieve yet another record in plasma $\beta$ up to $11 \%$. Equally important is the identification of the dominant modes as the plasma profiles were varied. Toroidally-induced Alfvén eigenmodes (TAE) and ballooning modes driven by energetic particles are of interest because they are predicted to cause alpha particle loss. The neutral beam current drive experiments on DIII-D with large $\epsilon \beta_{p}$ provide comprehensive testing of TAE mode theories. We have invested considerable effort in upgrading the GATO stability code to calculate the TAE mode spectrum. We can now use the code to study TAE modes in high $\beta$ elongated discharges such as those produced by DIII-D with high power neutral beam heating. 
In the transport area, we have accomplished the following:

- Brought new insights to the development of a first principle physical basis for the empirical energy confinement time scaling with a dimensionally similar scaling approach.

- Developed new and more realistic bifurcation models for the L-H transition and increased our understanding of divertor boundary layer physics and its connection to shear poloidal rotation.

Our theoretically motivated dimensionally-similar transport scaling experiment holds promise as a "wind-tunnel-like" method for scaling to ignition. These experiments will provide stimulus to new theories. Our understanding of the edge transport in a diverted plasma continued to expand with particular application to the Advanced Divertor Experiment. A simple theory which connects suppression of anomalous transport by velocity shear and standard neoclassical theory of poloidal rotation shows a number of features consistent with experimental observation for $\mathrm{L}-$ to $\mathrm{H}$-mode transition.

In the auxiliary heating area, we have accomplished the following:

- Continued to develop and upgrade quantitative tools to validate the physics of ECH and ICRF heating in tokamaks.

- Irivented techniques for using if to improve tokamak performance and diagnose plasma properties.

- Pioneered more efficient concepts to drive noninductive current.

Electron cyclotron heating is an importiant element of the DIII-D experimental program. Our codes routinely provide support by predicting and analyzing ley physics quantities such as absorption profile energetic electron distribution, and rf-driven current. Fast wave heating and current drive near the ion cyclotron frequency offers proven technique with readily available technology. Our theoretical work has provided a broad range of support for this experiment. Ion Bernstein wave heating was tried on DIII-D with mixed results and without significant heating. Several theoretical explanations were formed. The derivation of a general theory and the proposal of convincing physical pictures has solidified the understanding of wave helicity injection. 
We recall below the progrism plan which we submitted with our last proposal.

Areas in which we have made significant progress are noted with checkmarks. The details of our accomplishments can be found in the list of publications which accompanies this report. Suffice to point out here that we have achieved a majority of the tasks which we proposed back in FY89 at a funding level below what we were requesting.

\subsubsection{FY89-FY91 APP THEORY}

\subsubsection{MHD}

\section{$\boldsymbol{\beta}$ Enhancement}

Diverted Configurations

$\checkmark$ Establish $\beta$ scaling law in diverted configurations.

$\checkmark$ Study finite $n$ mode number stability of diverted configurations.

$\checkmark$ Study tearing mode stability of diverted configurations and its relation to "locked" mode.

$\checkmark$ Explore means for enhancement of $\beta$ limits.

Limiter Configurations

$\checkmark$ Establish detailed $\beta$ scaling laws for DIII-D plasmas.

$\checkmark$ Establish most unstable mode for DIII-D at high $\beta$.

Anisotropic Pressure

Calculate equilibria and high $n$ ballooning stability for anisotropic pressure profiles and in the presence of energetic particles.

\section{Disruptions:}

Low $\beta$ Disruptions

$\checkmark$ Establish ideal and resistive MHD stability boundaries for low $\beta$ DIII-D plasmas.

$\checkmark$ Compare with experimental data and optimize low $\beta$ discharges against disruptions. 
High $\beta$ Disruptions

$\checkmark$ Validate existing disruption scenarios against DIII-D data. Assess importance of sawtooth behavior for high $\beta$ disruptions.

$\checkmark$ Perform resistive MHD simulation of DIII-D discharges and disruptions. Further develop disruption scenarios.

\subsubsection{TRANSPORT}

\section{Transport Code Modeling of Experiments}

H-mode and Boundary Confinement Modeling

$\checkmark$ Do comparative L- and H-mode tests of drift wave models with DIII-D confinement data.

$\checkmark$ Develop H-mode divertor boundary layer model suitable for implementation in a transport code.

Confinement Analysis of Critical DIII-D ECH Heating Experiments

$\checkmark$ Analyze low density, high power ECH electron channel heating from ohmic phase to determine temperature dependence of the neo-Alcator component.

Analyze ECH edge heating experiments and attempt to distinguish models based on resistive interchange and moderate- $m$ tearing mode.

Numerical Simulation of Turbulent Transport in Tokamaks

Three Dimensional Global Simulations and Inhomogeneous Drift Wave Turbulence

$\checkmark$ Treat turbulence with better models for shear and curvature of magnetic field in both single and multiple helicity cases.

$\checkmark$ Investigate possible nonlocal effects.

Simulation of high $\beta$ ion temperature gradient mode turbulence.

$\checkmark$ Modify $3 \mathrm{D}(m, n, r)$ code to incorporate finite $\beta$ effects. 
Neoclassical and H-Mode Boundary Studies

Ion Transport Across a Separatrix

$\checkmark$ Calculate particle, energy, and momentum transport across separatrix with better collision and orbit models

$\checkmark$ Include fast ions in neoclassical theory for rotating plasmas and contributions to bootstrap current near a separatrix.

Neoclassically Consistent Microstability Theory

Establish appropriate neoclassical quasi-stationary equilibria including high power heating distortions from Maxwellian and study the influence on microstability.

\subsubsection{RF PHYSICS}

ECH:

Modeling of Heating and Current Drive

$\checkmark$ Utilize a complete ray-tracing, MHD and transport code package to model the DIII-D experiments.

$\checkmark$ Study properties of steady-state, noninductively driven current profiles.

RF Effects on Confinement

Quantify ECH induced radial and poloidal electric field and correlate with plasma rotation and confinement.

Study ECH in the open field line region of a diverted plasma.

Energetic Particle Physics

$\checkmark$ Continue upgrading absorption models at high power.

$\checkmark$ Develop 3 D Fokker-Planck code to quantify nonthermal electron effects.

ICRF

Modeling of Fast Wave and IBW Heating

$\checkmark$ Develop realistic antenna coupling code for $\mathrm{H}$-mode plasmas. 
$\checkmark$ Improve understanding of mode conversion processes and quantify energy deposition among particle specie.

Fasi Ion Physics

Calculate fast ion contribution to wave absorption and current drive.

$\checkmark$ Study ICRF interaction with microturbulence.

ICRF Induced Ion and Impurity Transport

$\checkmark$ Calculate ICRF induced particle and energy fluxes.

$\checkmark$ Study effect of localized $r f$ fields near antenna on impurity creation and transport. 


\subsection{HIGHLIGHTS OF FY91}

Technical progress has been made on a variety of topics. Synopses of these studies are summarized in our quarterly GA Theory News. A majority of the work has been submitted for publication in refereed journals as listed in the publications section and/or presented at various conferences. Three exemplary summary articles are included in this section.

Systematically and routinely assessing the stability to tearing modes in re:al axisymmetric non-circular tokamak equilibria with finite pressure is an important problem. During past year, a numerical method exploiting the formulation of Pletzier and Dewar [2-1] has been developed [2-2] (see 2.2.1). Recall that both resistive and slow-growth-rate MHD modes in axisymmetric toroidal systems have all their inertia concentrated in thin layers localized on resonant surfaces. Outside these layers, the mode structure is determined by ideal inertia-iree equations. Within the layers resistivity is important. Matching the solutions for the inner and outer layers determines the stability of the system. In systems with only one resonant layer per harmonic, the matching data is reduced to a single quantity well known as $\Delta^{\prime}$. However, real finite pressure systems break the poloidal symmetry and the matching data is contained in a matrix. An energy principle is normally used to evaluate ideal stability. The new work formulates an extended energy principle which incorporates the matching data from the ideal outer region together with the growth rate information from the inner region. This extended energy principle can be cvaluated in the same fashion as done in standard ideal MHD codes such as GATO, ERATO, and PEST. Thus, both resistive and ideal stability criterion can be obtained with a small modification on the existing codes.

A pressing issue in the confinement area is a better understanding of bulk plasma energy confinement and in particular its scaling with the operational parameters like power, density, current (or safety factor), and atomic mass. The latter two have been longstanding problems. During the past year some progress has been made on understanding the scaling with atomic mass [2-3] (see Subsection 2.2.2). The collisionless drift wave gyroBohm scaling is closest to explaining the L-mode scaling apart from is weak dependence on safety factor and atomic mass. It is weakly unfavorable to larger atomic mass contrary to the experimentally observed favorable mass dependence. However, previous heories have considered only pure plasmas. Adding an impurity species allows the mass ratio of the working gas and the impurity' to enter the problern so as to change the scaling with the working gas mass. A detailed application of quarilinear theory mixing length rules to the collisionless gyroBohm ion temperature gradient (ITG) mode turbulence has shown that a favorable mass scaling 
$\tau \propto A^{0.6}$ comparable to the empirical scaling can be obtained when an impurity species is included. Surprisingly, this result holds even for $Z_{\text {eff }}$ as low as 1.2.

Development of a practical diagnostic for the determination of the $\alpha$-particle spectra in fusing plasmas is a challenging problem and there are several proposals under consideration. Our expertise in rf physics has lead to a novel method using microwave scattering [2-4] (see Subsection 2.2.3). Scattering by electromagnetic waves by a plasma is mainly due to the electrons. However in the lower hybrid range of radio frequencies (LHRF), the scattering can be dominated by the collective effects of the ions. Previoue suggestions to extract information on the $\alpha$-particles have focused on the elec'rostatic approximation and the slow wave process. This, unfortunately, requires very high incident power levels for detectable scatter. The new method treats the electrornagnetic response and includes the fast wave. The latter is weakly damped by the electrons and ions, but strongly damped by the fast $\alpha$-particles. Detailed studies show that it should be possible to extract $\alpha$-particlee information from the collective resonant scattering off the fast mode.

During FY91, our theorists have made extended visits to TORE SUPRA, ASDEX, T-10, and JT-60 through the international collaboration program. In return, over twenty scientists from other institutions have visited the GA theory group. We organized a US/Japan Workshop on High- $\beta$ Plasma Our collaboration with UCSD plasma theorists continued to produce a number of important publications. 


\subsubsection{NUMERICAL STUDY OF A SINGULAR DIFFERENTIAL EQUATION RELEVANT FOR THE FINITE $\beta$ TEARING MODE IN A TOROIDAL PLASMA*}

The generalized Green's function method proposed by Miller and Dewar [1] and Pletzer and Dewar [2] for solving the singular differential equation occurring in the finite $\beta$ tearing mode problem has been tested numerically on a model differential equation. This method is compatible with a variational formulation of the problem and gives accurate numerical answers with high powers of convergence with respect to the number of grid points used. When the method is extended to the more physically relevant two-sided problem at moderate pressure gradients, a less stringent condition on the Frobenius expansion is required because the principal value of the otherwise divergent integrals associated with the method is shown to exist.

The central numerical problem of importance in the finite $\beta$ tearing mode in a toroidal plasma [3] is modeled on the solution of the following differential equation

$$
\mathcal{L} y=\left[\frac{d^{2}}{d x^{2}}-\frac{g(x)}{x^{2}}\right] y=0,
$$

near its singular point $x=0$. In (1), $g(x)=\sum_{i=0}^{\infty} x^{i} g_{i}$ is the power series expansion of $g(x)$ with $g_{i}$ the coefficient of the $i$-th power of $x$. The singular behavior is affected most by the values of $g_{0}$ and $g_{1}$.

The indicial equation $\alpha(\alpha-1)-g_{0}=0$ of the solution, $y=|x|^{\alpha} \sum_{j=0}^{\infty} a_{j} x^{j}$, determines two values for $\alpha=1 / 2 \mp \mu$, where $\mu=\left(g_{0}+1 / 4\right)^{1 / 2}$.

The interesting case for a tokamak is $g_{0}>0$, so we assume $\mu>1 / 2$. The $\alpha$ 's, in turn, determine the large and small solutions for $y$

$$
y_{L}=|x|^{1 / 2-\mu}\left[1+a_{1 L} x+\ldots+a_{j L} x^{j}+\ldots\right]
$$

and

$$
y_{S}=|x|^{1 / 2+\mu}\left[1+a_{1 S} x+\ldots\right] .
$$

*M.S. Chu, J.M. Greene, M. Klasky (Rensselaer Polytechnic Institute) and M.S. Chance (Princeton Plasma Physics Laboratory), General Atomics Report GAA20135 (1990), submitted to Journal of Computational Physics. 
The solution of the numerical problem is thus reduced to finding the value of $\Delta$, such that $y=y_{L}+\Delta y_{S}$ satisfies the boundary condition $y=0$ at $x= \pm 1$.

Much former numerical work has been expended on finding $\Delta$. One of the methods relies on a complicated sequence of convergence studies by using a sequence of finite elements to extract $\Delta$ [4]. The other one involves the shooting method [5], starting from $x=1$, relying on integrating the solutions accurately toward $x=0$ to extract the functional dependence of $y$ and the value of $\Delta$ from $y_{L}$ and $y_{S}$. These have been known to be satisfactory in one-dimensional problems where an extremely fine or adaptive mesh is easily implemented, but a straightforward extension to situations suitable for the two-dimensional problem is unclear.

An alternative method (generalized Green's function) has recently been proposed by Miller and Dewar [1] and Pletzer and Dewar [2]. It also utilizes the known behavior of the large and small solutions near $x=0$ and expresses $\Delta$ as an integral of the solutions. Due to the fact that $\Delta$ is expressed as an integral, this method is expected $t$ 's be superior.

The purpose of this work is to substantiate the findings of Refs. [1] and [2] and report that the method is directly applicable to possible situations arising from the finite $\beta$ tearing mode in a tokamak.

In the formulation of the generalized Green's function method, the solution $y$ is decomposed as $y \equiv y_{0}+y_{1}$, where $y_{1}$ is chosen as a sum of an $n$ term truncated representation of $y_{L}$ given by (2) and $y_{D}, y_{1}=y_{L}+y_{D}$. The component $y_{D}$ is $o\left(x^{\mu+1 / 2}\right)$ or, explicitly, $y_{D}=x^{i /}+a_{D 1} x^{h+1}+\ldots$. It represents the "deviation" of $y_{1}$ from $y_{L}$, and $h$ is required to be larger than $\mu+1 / 2$ so that the deviation is smaller than the small solution near $x=0, y_{1}$ is also required to satisfy the boundary condition, $y_{1}(1)=0$. Wi h $y_{1}$ thus specified, $y_{0}$ is determined by the relationship

$$
\mathcal{L} y_{0}=-\mathcal{L} y_{1}
$$

with the boundary conditions $y_{0}(0)=y_{0}(1)=0$. We showed that, in this case, $\Delta$ is given by

$$
\mu \Delta(a, b)=I_{1}(a, b)+I_{2}(a, b)-I_{3}(a, b)
$$

where $a=0, b=1$, and 


$$
\begin{aligned}
& I_{1}(a, b)=\frac{1}{2} \int_{a}^{b} y_{1} \mathcal{L} y_{1} d x \\
& I_{2}(a, b)=\int_{a}^{b} y_{0} \mathcal{L} y_{1} d x, \\
& I_{3}(a, b)=\frac{1}{2} \int_{a}^{b}\left[\left(\frac{d y_{0}}{d x}\right)^{2}+\frac{g}{x^{2}} y_{0}^{2}\right] d x .
\end{aligned}
$$

It is easy to verify that $\Delta$ given by (5) is variational with respect to $y_{0}$ with (4) as its Euler equation for the eigenfunction $y_{0}$. The solution $y_{0}$ gives $I_{2}=2 I_{3}$. Furthermore, (5) is invariant to the choice of $y_{D}$ so long as $h>\mu+1 / 2$. The variational expression given by (5) is a desirable form to be used for the tearing mode study because, in this case, $I_{3}$ corresponds to $\delta W$ and $\mathcal{L}$ corresponds to the Euler equation operator [6]. Both $\delta W$ and $\mathcal{L}$ are important concepts in magnetohydrodynamics.

A finite element computer program employing Hermite cubics has been constructed to solve the variational problem for $\Delta$. Three-point Gaussian integration was used for the evaluation of the coefficient matrix in the variational problem and the integrals $I_{1}, I_{2}, I_{3}$. Mesh accumulation is arranged by taking the mesh points $x_{i}$ to be given by $x_{i}=(i-1)^{\alpha} \delta x$. For $0<g_{0}<0.75$, or $1 / 4<\mu<1$, the convergence of $I_{1}$ requires the Frobenius expansion (2) of $y_{L}$ be carried out at least to include the linear term $a_{1 L}$. To anticipate situations in the finite $\beta$ tearing mode in which multiple singular surfaces may arise, say at $x=a<1$, we modify $y_{D}$ so that $y_{1}(x)=0$ for $a<x<1$. Thus, the numerical study consists in testing the accuracy of $\Delta$ for different $g_{0}, g_{1}$, with respect to the variation in the number of terms in the Frobenius expansions and different values of $h, a$, and $\alpha$.

Three cases (a) $g=g_{0}$, (b) $g=g_{0}-x$, and (c) $g=g_{0}+x$ in which analytic solutions are known are chosen for comparison with the numerical code. In the numerical study, the number of terms used in the Frobenius expansion has been varied. It is verified that only two terms are needed. The ranges of $g_{0}, h, a$, and $\alpha$ used are $0<g_{0}<1, \mu+1 / 2<h<5,0<a<1$, and $1<\alpha<5$. The maximum number of grid points used has been varied up to 200 .

A typical result of the code is shown in Fig. 1 for the case where $g=g_{0}-x$. In this case, $\Delta$ has the analytic value

$$
\frac{\pi}{2 \Gamma(2 \mu)^{2}}\left[\frac{Y_{2 \mu}(2)}{J_{2 \mu}(2)}-\frac{\cos (2 \mu \pi)}{\sin (2 \mu \pi)}\right],
$$


(a)

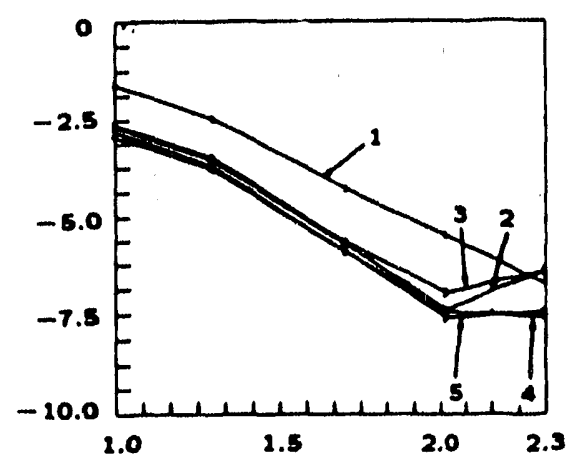

(b)

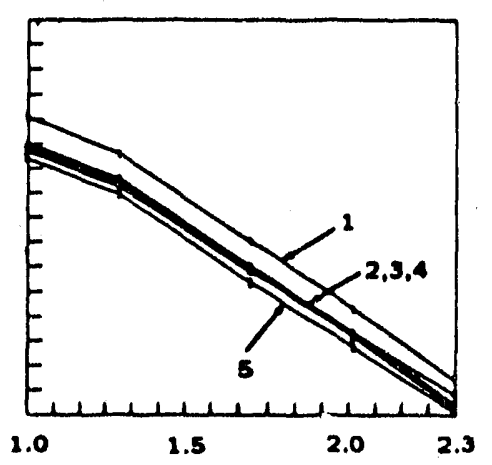

(c)

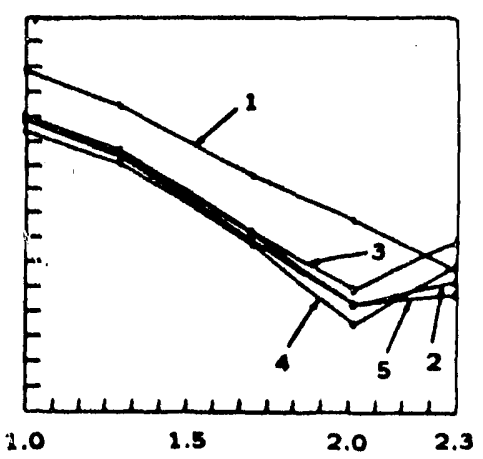

FIG. 1. Plot of the magnitude of (a) relative total error, (b) relative $I_{1}$ error, and (c) relative $I_{2}$ error in $y_{0}$ as a function of number of grid points $N$ employed in the numerical c nputation for a case in which $g=g_{0}-x$. In this example, $\alpha=4$ and $h=3.5$. The value of $g_{0}$ for curve 1 is $0.01 ; 2,0.17 ; 3,0.35 ; 4,0.62 ;$ and $5,0.74$. Note that the abscissa and the ordinate are on $\log _{10}$ scale.

where $Y$ and $J$ are the modified Bessel functions. Shown in Fig. $1(a)$ is the relative error in $\Delta\left(\varepsilon_{\Delta}\right)$ with respect to the number of grid points used; in (b), the relative error in $I_{1}\left(\varepsilon_{I_{1}}\right)$; and in (c), the the relative error in $I_{2}\left(\varepsilon_{I_{2}}\right)$. Note the log-log scales used. It is clearly seen that all the relative error all scaled as $N^{-6}$. In this example, $\varepsilon_{I_{2}}$, and consequently reduces until the number of grid points reaches 100 and then increases due to truncation error coming in from the numerical evalustion of other quantities such as $Y_{2 \mu}$ and $\Gamma(2 \mu)$. This is checked by increasing the grid points beyond 200 and observe that the error further increases. The value of $a$ used here is 0.5. Extensive computation for other cases with modified support $a$ of the large solution gives essentially the same behavior as shown in Fig. 1.

The above problem may be extended to cover the interval $(-1,1)$, for a combined $\Delta$ defined to be $\Delta(-1,1)=\lim _{\varepsilon \rightarrow 0}[\Delta(-1,-\varepsilon)+\Delta(\varepsilon, 1)]$. In this case, $I_{1}$ may be defined as the principal value of a convergent integral and only one term in the Frobenius expansion is needed.

In conclusion, we have found that the generalized Green's function method proposed by Dewar et al. $[1,2]$ allows a variational formulation of the tearing mode problem in finite $\beta$. With sufficiently high grid packing factor $\alpha \simeq 4$, the method is accurate. The requirement of internal boundary points increases the magnitude of the relative error but keeps the correct scaling with the grid number to $N^{-6}$. For t? range of $0<g_{0}<0.75$, only one term in the Frobenius xpansion is needed when only the sum of the $\Delta$ from both sides of the singularity is desired. 
We note, in particular, the generalized Green's function method, by matching the large "trial function" to the large solution with sufficient accuracy near the singularity, renders the final answers in terms of convergent integrals of the solutions over the entire interval. For $2 \mathrm{D}$ problems, this is superior to the method used in Ref. 4, where only the finite element closest to the singular surface is forced to match to the large solution, resulting in numerical difficulty.

It is also instructive to compare the generalized Green's function method with the method used in Ref. 5. The two methods are similar at the start. Both methods seek to understand the analy tic behavior of the large and small solutions by performing Frobenius expansion of the equations. The difference lies in the utility of this analytic information in the two methods. The method in Ref. 5 then went on to use purely numerical means to extract the coefficients of both the large and small solutions. An adaptive grid in the radial direction (excluding the singular point!), was actually required to obtain these coefficients. In the generalized Green's function method, the analytic development is carried further. The analytic behavior of the singular solutions was used and folded into numerically convergent integrals covering the whole radial interval. Only the small solution needs to be handled explicitly by numerical means. We regard these conceptual features in the generalized Green's function method superior to that given in Ref. 5. These are explicitly relevant in 2D situations. The major difficulty in extending the generalized Green's function method to $2 \mathrm{D}$ is that the analytic pre-development of the theory leading to the large solution could be quite formidable.

This is a report of work sponsored by the U.S. Department of Energy under Contract Nos. DE-AC03-89ER53277 and DE-AC02-76-CHO-3073.

\section{References}

[1] A.D. Miller and R.L. Dewar, J. Comput. Phys. 66 (1986) 356.

[2] A. Pletzer and R.L. Dewar, in Computational Techniques \& Applications: CTAC89 (W.L. Hogarth and B.J. Noye, Eds.) (Proc. Int. Conf. Brisbane, Australia, July 10-12, 1989), Hemisphere, New York, 1990, 429.

[3] R.L. Dewar and R.G. Grimm, Singular Finite Element Methods in Plasma Stability Computations - a Simple Model, in Computational Techniques and Applications: CTAC-83, (B.J. Noye and C. Fletcher, Eds.) North Holland, Amsterdam, 1984, 730.

[4] R.C. Grimm, R.L. Dewar, J. Manickam, S.C. Jardin, A.H. Glasser, and M.S. Chance, in Plasma Physics and Controlled Fusion Research (Proc. 9th Int. Conf. Baltimore, 1982) IAEA, Vienna, 1983, Vol. 3, p. 35. 
APP REPORT TO DOE, FY89 THROUGH FY91

[5] M.S. Chance et al., Nucl. Fusion 22 (1982) 187.

[6] M.S. Chu, M.S. Chance, J.M. Greene, and T.H. Jensen, Phys. Fluids B 2 (1990) 97. 


\subsubsection{ISOTOPIC SCALING IN THE DRIFT WAVE MODEL*}

Impurity ion dynamics are incorporated into a numerical stability analysis of the electrostatic ion temperature gradient (ITG) mode in a sheared slab model. It is found that in hydrogen and deuterium plasmas growth rates are inversely proportional to primary ion mass when $Z_{\text {eff }}<2$; for $Z_{\text {eff }} \geqslant 2$, the ITG mode is generally stable. Using quasilinear theory, it is also shown that ITG mode turbulence in in hydrogen and deuterium plasmas in the low $Z_{\text {eff range (typical of high density ohrnic plasmas) }}$ obtains energy confinement time scaling with atornic mass number $A$ of the primary ion species; $\tau_{\mathrm{E}} \propto A^{\beta}$ with $\beta \simeq 0.6$. Conversely, the present analysis indicates that confinement in helium plasmas is similar to the results for hydrogen.

Anomalous transport in tokamaks is widely believed to be driven by a combination of trapped electron and ion temperature gradient (ITG) mode turbulence in the bulk plasma [1]. Based on this model, reasonably good agreement has been obtained in detailed comparisons between theory and experiment for both low and high density ohmically heated and auxiliary heated discharges [2-5]. Generally, the theoretical transport models are based on the assumption of pure plasmas, with little consideration given to the role of impurities in transport processes. One of the unresolved problems for this interpretation of anomalous energy transport is the absence of any appreciable atomic mass scaling in the predicted energy confinement time. Indeed, in nearly all pure plasma drift wave transport models, the ion mass never explicitly appears. However, with the introduction of a second ion species (of different mass), the impurity ions, the primary ion atomic mass becomes an explicit parameter in the drift wave transport model.

Here we show that in certain circumstances impurity ion effects can lead to qualitatively different stability properties for the ITG mode than in pure plasmas, introducing explicit primary ion mass dependence. Further, quasilinear theory predicts an appreciable atomic mass scaling of energy confinement time between hydrogen and deuterium (approximately $\tau_{\mathrm{E}} \propto A^{0.6}$ ) for ITG mode turbulence in the low $Z_{\text {eff range, }}$ $Z_{\text {eff }}<2$, typical of high density ohmic plasmas. Interestingly, the present analysis also indicates that confinement in helium plasmas is, at most, only comparable to hydrogen plasmas.

*R.R. Dominguez, General Atomics Report GA-A20184 (1991), submitted to Nuclear Fusion. 
To illustrate the relevant impurity ion modifications, we restrict consideration to the sheared slab model. Toroidal coupling should not qualitatively change the present conclusions. The perturbed density responses to the electrostatic perturbation $\delta \Phi$ for the electrons and impurity ions, assumed fluid-like $k_{\perp} a_{\mathrm{I}} \ll 1, \omega \gg k_{\|} v_{\mathrm{TI}}$, are given by

$$
\delta r_{e}=\frac{n_{e} e}{T_{e}} \delta \Phi\left(1-i \delta_{e}\right)
$$

and

$$
\begin{aligned}
\delta n_{\mathrm{I}}=- & \frac{Z_{\mathrm{I}} n_{\mathrm{I}} e}{T_{\mathrm{I}}} \delta \Phi \\
& \times\left\{\frac{\omega_{\mathrm{I}}^{*}}{\omega}+\left(\frac{1}{2} k_{\perp}^{2} a_{\mathrm{I}}^{2}-\frac{1}{2} \frac{k_{\|}^{2} v_{\mathrm{TI}}^{2}}{\omega^{2}}\right)\left[1-\frac{\omega_{\mathrm{I}}^{*}}{\omega}\left(1+\eta_{\mathrm{I}}\right)\right]\right\},
\end{aligned}
$$

where $\delta_{e}$ is the model non-adiabatic electron factor, $n_{e}$ and $n_{I}$ are the electron and impurity ion densities, $\omega_{\mathrm{I}}^{*}$ is the impurity ion diamagnetic drift frequency, $a_{\mathrm{I}}$ is the impurity ion gyroradius, $Z_{\mathrm{I}}$ is the impurity ion charge, and $\eta_{\mathrm{I}}=\partial \ell_{\mathrm{n}} T_{\mathrm{I}} / \partial \ell_{\mathrm{n}} n_{\mathrm{I}}$. The perturbed density response for the primary ions is fully kinetic [6]

$$
\begin{aligned}
\delta n_{\mathrm{i}}=- & \frac{Z_{\mathrm{i}} n_{\mathrm{i}} e}{T_{\mathrm{i}}} \delta \Phi\left(1+\left\{\left(1-\frac{\omega_{\mathrm{i}}^{*}}{\omega}\right) \Gamma_{0}+\frac{1}{2} \eta_{1} \frac{\omega_{\mathrm{i}}^{*}}{\omega}\left[\Gamma_{0}-2 b_{\mathrm{i}}\left(\Gamma_{1}-\Gamma_{0}\right)\right]\right\} \lambda_{\mathrm{i}} Z\left(\lambda_{\mathrm{i}}\right)\right. \\
& \left.-\eta_{\mathrm{i}} \frac{\omega_{\mathrm{i}}^{*}}{\omega} \Gamma_{0} \lambda_{\mathrm{i}}^{2}\left[1+\lambda_{\mathrm{i}} Z\left(\lambda_{\mathrm{i}}\right)\right]\right),
\end{aligned}
$$

where $Z_{i}$ is the primary ion charge, $n_{i}$ is the primary ion density, $\omega_{i}^{*}$ is the primary ion diamagnetic drift frequency, $\eta_{\mathrm{i}}=\partial \ell \mathrm{n} T_{\mathrm{i}} / \partial \ell \mathrm{n} n_{\mathrm{i}}, Z\left(\lambda_{\mathrm{i}}\right)$ is the plasma dispersion function, $\lambda_{i}=\omega /\left|k_{11}\right| v_{T_{1}}, \Gamma_{0,1}=I_{0,1} \exp \left(-b_{i}\right), I_{0,1}$ are modified Bessel functions of argument $b_{1}$, and $b_{1}=k_{\perp}^{2} a_{1}^{2} / 2$ with $a_{i}$ the primary ion Larmor radius.

The procedure for obtaining the radial eigenmode equation from Eqs. (1a) through (1c) is well known [6]; $k_{\perp}^{2} \rightarrow k_{\theta}^{2}-\partial^{2} / \partial x^{2}$, with a Taylor series expansion of the Bessel functions about $k_{\perp}=k_{\theta}$. This obtains the differential equation

$$
\begin{aligned}
& \left(\frac{\partial^{2}}{\partial y^{2}}-\left\{1-i \delta_{\mathrm{e}}+\tau(1-\mu) Z_{\mathrm{i}} Q_{\mathrm{i}}-\mu \frac{L_{n_{\mathrm{e}}}}{L_{n_{\mathrm{I}}}}\left(\frac{\omega_{\mathrm{e}}^{*}}{\omega}\right)\right.\right. \\
& \left.+\mu \tau\left(\frac{b_{\mathrm{i} \theta} Z_{\mathrm{i}}^{2}}{\alpha}-\frac{\alpha}{2 \lambda_{\mathrm{i}}^{2}}\right)\left[1-\frac{\omega_{\mathrm{I}}^{*}}{\omega}\left(1+\eta_{\mathrm{I}}\right)\right]\right\} / \\
& \left.Z_{\mathrm{i}}\left\{Z_{\mathrm{i}} \frac{\mu}{\alpha}\left[1-\frac{\omega_{\mathrm{I}}^{*}}{\omega}\left(1+\eta_{\mathrm{I}}\right)\right]+(1-\mu) \frac{\partial Q_{\mathrm{i}}}{\partial b_{\mathrm{i} \theta}}\right\}\right) \delta \Phi=0,
\end{aligned}
$$


where $Q_{1}$ is defined in Eq. (1c), $\delta n_{1}=-\left(Z_{1} n_{1} e / T_{1}\right) Q_{1} \delta \Phi, b_{1 \theta}=k_{\theta}^{2} a_{1}^{2} / 2, \tau=T_{e} / T_{1}$, and $y=x / \rho_{\mathrm{s}}\left(\rho_{\mathrm{s}}=c_{\mathrm{s}} / \Omega_{\mathrm{i}}\right.$ where $c_{\mathrm{s}}$ is the sound speed and $\Omega_{\mathrm{l}}$ is the primary ion gyrofrequency). This resulting eigenmode differential equation is solved for the complex eigenvalues $\omega$ with 2 "shooting" code, subject to the boundary conditions for even modes, $\delta \Phi^{\prime}(0)=\delta \Phi(\infty)=0$; odd modes are generally more stable and not considered here. All frequencies are normalized to $\omega_{\mathrm{a}}=c_{\mathrm{s}} / L_{n_{\mathrm{e}}}$ with $c_{\mathrm{B}}=\left(T_{\mathrm{e}} / m_{\mathrm{i}}\right)^{1 / 2}$, and lengths are normalized to $\rho_{\mathbf{s}}$. The impurity ion effects are characterized by the parameters $\mu=\left(Z_{\text {eff }}-Z_{1}\right) /\left(Z_{I}-Z_{1}\right), \alpha=Z_{\mathrm{I}} m_{\mathrm{I}} / m_{\mathrm{I}}\left(m_{\mathrm{I}}\right.$ is the impurity ion mass), and $L_{n_{\mathrm{e}}} / L_{n_{\mathrm{I}}}$. Unless otherwise noted, we shall take $L_{n_{e}} / L_{n_{\mathrm{I}}}=1$. It is also assumed throughout that $Z_{\mathrm{r}}=6$ (carbon) so that $\alpha=0.5,1.0$, and 2.0 correspond to primary ion species of hydrogen $\left(Z_{1}=1\right)$, deuterium $\left(Z_{1}=1\right)$, and helium $\left(Z_{1}=2\right)$, respectively. In the numerical solutions, we also fix the shear length as $L_{n_{\mathrm{e}}} / L_{\mathrm{s}}=0.1$, the electron temperature gradient paramete $\eta_{\mathrm{e}}=2$, and assume that the temperature gradient scale lengths for electrons and primary ions are equal.

The ITG mode is thought to be the dominant anomalous loss mechanism in the bulk plasma in both high density ohmic plasmas as well as auxiliary heated plasmas $[2,3]$. In high density ohmic plasmas, the impurity concentration is generally small, $Z_{\text {eff }}<2$ [7], while in auxiliary heated plasmas $Z_{\text {eff }}$ tends to be larger, particularly in $\mathrm{H}$-mode. In the numerical solutions of the ITG mode discussed here, the electron response is assumed adiabatic $\left(\delta_{e}=0\right)$ and we take $\tau=1$ and $\mu=0.1$ (unless otherwise noted), typical of high density ohmic plasmas. In pure plasmas, the ITG mode tends to be fluid-like Rew $\simeq$ Imw, and the Weber approximation obtains reasonably good agreement with the full numerical solutions [6]. However, with the introduction of impurity ions, the growth rates in hydrogen and deuterium ( $\alpha=0.5$ and 0.1 , respectively) are substantially reduced, while the real frequencies are only slightly affected. This indicates a significant shift to a resonant-type instability.

The dependence of the impurity modified ITG mode on the mass parameter $\alpha$ is shown in Fig. 1. We fix $\mu=0.1\left(Z_{\text {eff }}=1.5\right.$ for $\alpha=0.5$ and $1.0, Z_{\text {eff }}=2.4$ for $\alpha=2.0$ ) and plot the real and imaginary part of $\omega$ for the fundamental harmonic versus $b_{1 \theta}$ for $\alpha=0.5,1.0$, and 2.0. Again, the real frequency [Fig. 1(a)] has weak dependence on $\alpha$ for $\alpha=0.5,1.0$, and 2.0. More interestingly, though, the growth rate [Fig. 1(b)] shows an inverse dependence on $\alpha$ between $\alpha=0.5$ and 1.0, but $\alpha=2.0$ (and $Z_{\mathrm{i}}=2$ ) is nearly identical to the $\alpha=0.5$ curve. For fixed impurity species, this $\alpha$ between $\alpha=0.5$ and 1.0 scaling is equivalent to a primary ion mass scaling. The half-widths $\Delta x / \rho_{\mathrm{s}}$ of the eigenfunctions for these cases also show an inverse $\alpha$ dependence, although it is weak (of order $\alpha^{-0.2}$ ). For pedagogical purposes, we also 
(a)
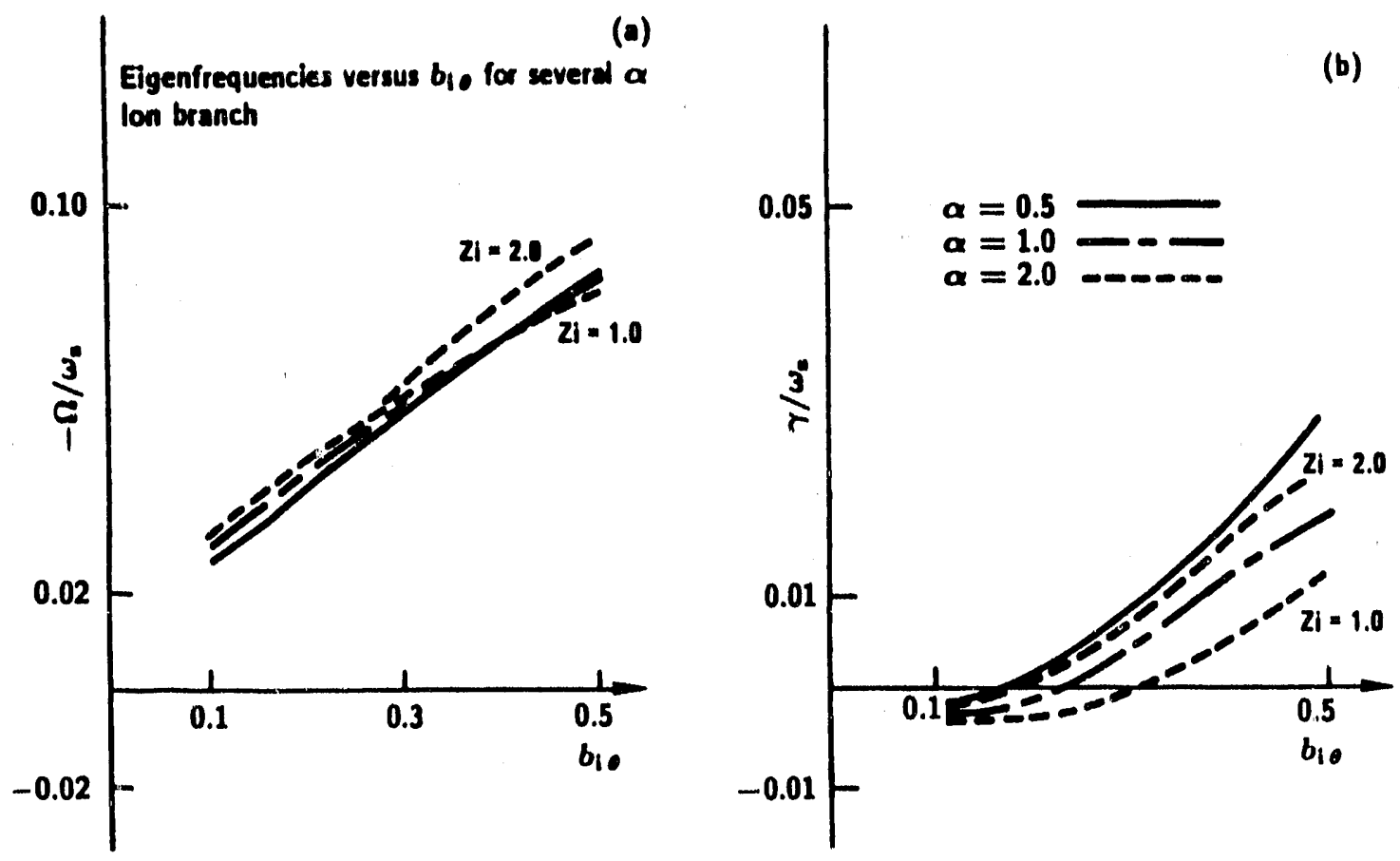

FIG. 1. (a) The real frequency of the ITG mode versus $b_{1_{\theta}}$ for several $\mu$ and $\alpha=1$; ( $)$ growth rate of the ITG mode versus $b_{1_{\theta}}$ for several $\mu$. In the figure, $\tau=1, \eta_{\mathrm{l}}=\eta_{\mathrm{I}}=2, L_{n_{\mathrm{e}}} / L_{\mathrm{s}}=0.1$, $L_{n_{\mathrm{e}}} / L_{n_{\mathrm{I}}}=1.0$, and $\delta_{\mathrm{e}}=0$.

show in Fig. 2 the results for the fictitious case $\alpha=2, Z_{i}=1$ in order to isolate the effect of primary ion charge on the stability of the ITG mode.

The quasilinear ion energy transport equation is given by

$$
\frac{3}{2} \frac{\partial}{\partial t}\left(n_{\mathrm{i}} T_{1}\right)+\frac{\partial}{\partial x} Q_{x}^{(\mathrm{i})}=\Delta
$$

where $Q_{\mathrm{x}}^{(\mathrm{i})}$ is the radial flux of ion energy and $\Delta$ is an anomalous energy exchange term (generally small). The radial ion heat conduction flux $q_{x}^{(i)}$ is related to $Q_{x}^{(i)}$ by $q_{x}^{(i)}=Q_{x}^{(i)}-\frac{5}{2} \Gamma_{x}^{(i)} T_{i}$ where $\Gamma_{x}^{(i)}$ is the radial flux of primary ions; note that for a multi-ion species plasma, $\Gamma_{x}^{(i)} \neq \Gamma_{x}^{(e)}$. In the present analysis, convective loss is ignored. The quasilinear expression for $q_{x}^{(i)}$ is given by [6]

$$
q_{x}^{(1)}=Z_{\mathrm{i}} n_{\mathrm{i}} T_{1} L_{n_{\mathrm{e}}} \int \frac{d k_{\theta}}{2 \pi} \omega_{\mathrm{e}}^{*} W_{k_{\theta}} \mathcal{H}_{\mathrm{i}}^{(2)}
$$

where 
(a)

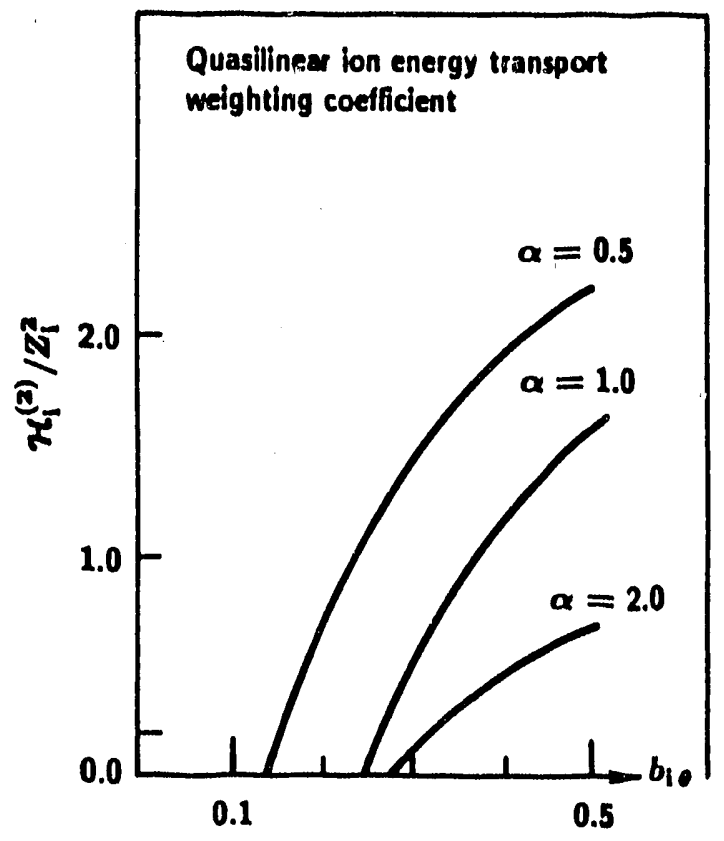

(b)

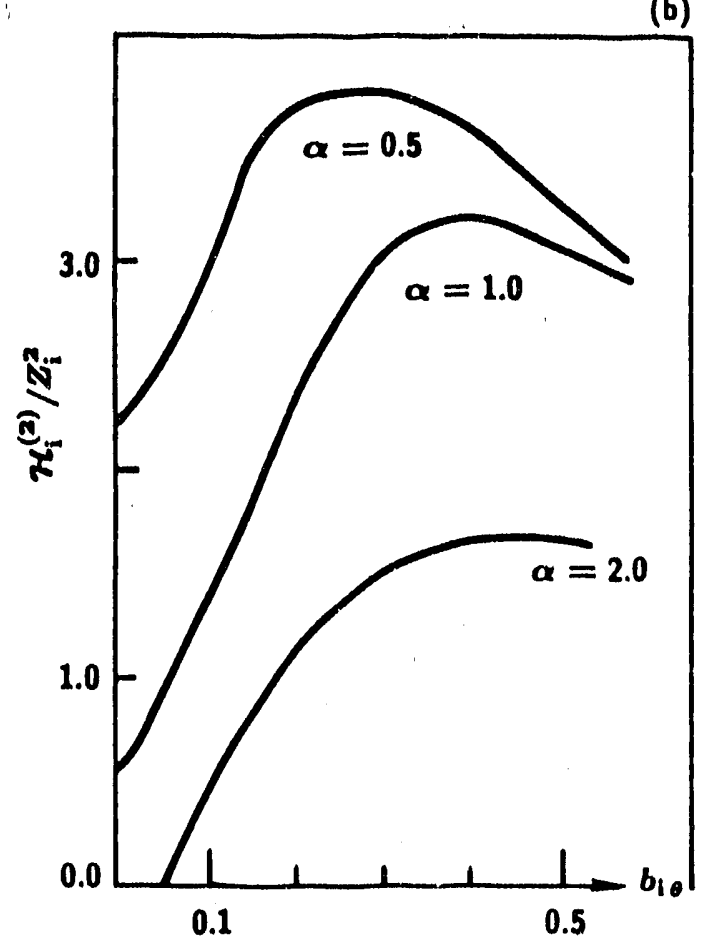

FIG. 2. (a) The real frequency of the ITG mode versus $b_{\mathrm{i}_{\theta}}$ for several $\alpha$ and $\mu=0.1$; (b) growth rate of the ITG mode versus $b_{\mathrm{i}_{\theta}}$ for several $\alpha$. The same parameters as in Fig. 1 are used with $\mu=0.1$.

$$
W_{k_{\theta}}=\int_{-\infty}^{+\infty} d x\left|\frac{e \delta \Phi(x)}{T_{e}}\right|^{2},
$$

and $\mathcal{H}_{\mathrm{i}}^{(2)}$ is a spatially averaged weighting function given by

$$
\mathcal{H}_{\mathrm{i}}^{(2)}=\operatorname{Im}\left\{\frac{\int d x|\delta \Phi(x)|^{2} H_{\mathrm{i}}^{(2)}(x)}{\int d x|\delta \Phi(x)|^{2}}\right\} .
$$

The function $H_{1}^{(2)}(x)$ is a very complicated expression involving $\omega$, the plasma dispersion function, and the functions $\Gamma_{0,1}=I_{0,1} \exp \left(-b_{i}\right)[6]$. Quasilinear theory does not provide an absolute magnitude, or parametric dependence, for the intensity factor $W_{k_{\theta}}$ in Eq. (5). However, here we are interested in establishing the primary ion mass dependence of $q_{x}^{(i)}$ (manifest through the factor $\alpha$ ). The half-widths of the eigenfunctions $\delta \Phi(x)$ scale approximately as $\alpha^{-0.2}$ for $\alpha=0.5$ and 1.0 which combined 
with the mixing length estimate for the saturated amplitude determines the $\alpha$ scaling of $W_{k_{\theta}} ;$ for $\alpha=2, \delta \Phi(x)$ has a somewhat larger width than $\alpha=0.5$. ITG mode turbulence exhibits an inverse cascade in $k_{\theta}$ [8], and consequently we assume that $W_{k_{\theta}}$ peaks at smaller $b_{1 \theta}$ than the linear growth rates $\left(b_{1 \theta} \simeq 0.5\right)$. It remains to examine the dependence of $\mathcal{H}_{\mathrm{i}}^{(2)}$ on $\alpha$. For $\mu=0.1\left(Z_{\text {eff }}=1.5\right.$ for $\alpha=0.5$ and 1.0, $Z_{\text {eff }}=2.4$ for $\alpha=2.0$ ), this result is shown in Fig. $2(\mathrm{a})$, where $\mathcal{H}_{\mathrm{i}}^{(2)} / Z_{1}^{2}$ is plotted versus $b_{1 \theta}$ for $\alpha=0.5,1.0$, and 2.0. The quantity $\mathcal{H}_{1}^{(2)} / Z_{1}^{2}$ is shown since, based on the mixing length estimate of saturated amplitude, it exhibits the sirrongest explicit $Z_{1}$ dependence in Eq. (4).

The cases shown in Fig. 2 (a) are obtained from a numerical evaluation of the $\mathcal{H}_{1}^{(2)}$ function using the eigenfrequencies and eigenfunctions corresponding to Fig. 1. Systematic studies of high density ohmic hydrogen and deuterium plasmas in ASDEX [9] have reported significant isotope effects, even with trace impurity ion levels, $Z_{\text {eff }}-1 \ll 1$. In Fig. 2 (b), we show a result analogous to Fig. 2 (a), except $\mu=0.04\left(Z_{\text {eff }}=1.2\right.$ for $\alpha=0.5$ and $1.0, Z_{\text {eff }}=2.16$ for $\left.\alpha=2.0\right)$. At this lower $Z_{\text {eff }}$, the normalized $\alpha=2$ quasilinear weighting coefficient is somewhat larger. In the range $0.1<b_{1 \theta}<0.3, \mathcal{H}_{\mathrm{i}}^{(2)} / Z_{1}^{2}$ on average is approximately proportional to $\alpha^{-1}$ for hydrogen, deuterium, and helium. In Fig. $2(\mathrm{a})$ and (b), the $\alpha=0.5$ and 1.0 dependence is substantially stronger than the $\alpha$ dependence of the corresponding linear growth rates and, conversely, the $\alpha=2.0$ is somewhat weaker than in the linear growth rates. Indeed, $\mathcal{H}_{\mathrm{i}}^{(2)}$ can be large even for marginally stable modes.

It is straightforward to deduce the mass scaling of confinement time assuming that the saturated amplitude is peaked at $b_{\mathrm{i} \theta}$ smaller than the $b_{\mathrm{i} \theta}$ for the maximum linear drive, $\chi_{\mathrm{i}} \propto\left(\Delta x / \rho_{\mathrm{s}}\right)^{2} \omega_{\mathrm{s}} \bar{\rho}_{\mathrm{s}}^{2}\left[\mathcal{H}_{\mathrm{i}}^{(2)} / Z_{\mathrm{i}}^{2}\right]$ where $\bar{\rho}_{\mathrm{s}}$ is $\rho_{\mathrm{s}}$ evaluated at $Z_{1}=1$ and $\mathcal{H}_{1}^{(2)} / Z_{1}^{2}$ is evaluated at the peak of the saturated spectrum. The energy confinement time $\tau_{\mathrm{E}}=a^{2} / \chi$ is equated to the energy replacement time, which is proportional to $n_{\mathrm{e}} T_{\mathrm{c}} a^{2} R / P$ ( $P$ is the total input power). For ohmic heating, $P$ is proportional to $T_{e}^{-1.5}$. Using a mixing length estimate for the saturated mode amplitude in $W_{k_{\theta}}$ $\left(W_{h_{\theta}} \propto \alpha^{0.8} / Z_{1}^{0.4}\right)$, and the mass scaling of $\mathcal{H}_{1}^{(2)}$, we deduce that $\chi \propto T_{\mathrm{e}}^{1.5} Z_{1}^{1.8} / A^{0.8}$ for ITG mode turbulence ( $A$ is the atomic mass number and $Z_{\mathrm{i}}$ the charge of the primary ion species). Thus, for high density ohmic plasmas where ITG turbulence dominates, we conclude that $\tau_{\mathrm{E}} \propto A^{0.58}$ for hydrogen and deuterium plasmas; for helium plasmas, quasilinear theory predicts that $\chi$ is comparable to hydrogen, with $\tau_{\mathrm{E}} \propto A^{0.56} / Z_{\mathrm{i}}$ and any additional improvement from larger $A$ being offset by the larger $Z_{1}$ of a helium plasma. The results for hydrogen and deuterium compare favorably with the results from the ASDEX study [9] which deduced that $\tau_{\mathrm{E}} \propto A^{0.5}$ in the high density ohmic region. 
This is a report of work sponsored by the U.S. Department of Energy under Contract No. DE-AC03-89ER53277.

\section{Reference:}

[1] R.E. Waltz, R.R. Dominguez, F.W. Perkins, Nucl. Fusion 29 (1989) 351.

[2] R.R. Dominguez, R.E. Waltz, Nucl. Fusion 27 (1987) 65.

[3] M.H. Redi, W.M. Tang, P.D. Efthimion, et al., Nucl. Fusion 27 (1987) 2001.

[4] A. Rogister, G. Hassenberg, F. Waelbroeck, J. Wieland, Nucl. Fusion 28 (1988) 1053.

[5] D.L. Brower, M.H. Redi, W.M. Tang, et al., Nucl. Fusion 29 (1989) 1247.

[6] R.E. Waltz, W. Pfeiffer, R.R. Dominguez, Nucl. Fusion 20 (1980) 43.

[7] D.P. Schissel, R.E. Stockdale, H. St. John, W.M. Tang, Phys. Fluids 31 (1988) 3738.

[8] S. Hamaguichi, W. Horton, Phys. Fluids B 2 (1990) 1833.

[9] F. Wagner, M. Bessenroth-Weberpals, H.U. Fahrbach, et al., 16th Euro. Conf. on Controlled Fusion and Plasma Heating, Venice (1989), 13B, part 1, 195. 


\title{
2.2.3. ELECTROMAGNETIC EFFECTS IN FLUCTUATIONS OF NON-EQUILIBRIUM PLASMAS AND APPLICATION TO ALPHA-DIAGNOSTICS*
}

\begin{abstract}
Although normally significant density fluctuations occur in only electrostatic modes, the fast mode in the lower hybrid range of frequencies gives an exceptional example in which the mode is non-trivially electromagnetic, and yet the density fluctuations are comparable to the slow mode. This fact has important implications on the scattering of electromagnetic waves by non-equilibrium plasmas such as fusionplasmas with energetic $\alpha$-particles. It makes microwave scattering a more plausible scheme for $\alpha$-diagnostics.
\end{abstract}

The scattering of electromagnetic waves by a plasma offers a powerful diagnostic tool [1-4]. Recently, there is interest in using microwave scattering for $\alpha$-diagnostics in fusion plasmas [5-9]. As is well known, the scattering of electromagnetic waves by a plasma is mainly due to the electron density fluctuations. However, in the lower hybrid range of frequencies (LHRF), the electron fluctuation spectrum may be dominated by the collective effects due to the ions. Scattering of microwaves by fluctuations in this spectral range can thus give valuable information about ions. The scattering cross-section is usually calculated in the electrostatic approximation [5-9]. In LHRF, this picks up the slow wave resonance scattering, and as shown in Refs. 7 and 8 , the contribution to the density fluctuations is usually dominated by electron or thermal ions. In order to extract information about energetic $\alpha$-particles, it appears preferable to measure the scattered wave off-resonance. This considerably reduces the scattered amplitude, and thus demands a much higher incident microwave power.

In this paper, we would like to point out that in LHRF, the fast mode (magnetosonic) presents an exceptional case in which the mode is electromagnetic and yet the fluctuation amplitude is still comparable to the electrostatic slow mode. This is because in LHRF, although one cannot obtain the fast mode from the electrostatic approximation, the dominant component of the field is, nevertheless, the electrostatic component. This has important consequences in applications to $\alpha$-diagnostics because fast waves exist in a wider frequency range than the slow waves, and more importantly, the damping of the fast waves by electrons and thermal ions is usually much weaker than in the slow wave case, while the damping due to energetic particles remains quite strong. It thus appears more plausible to extract $\alpha$-particle information from the collective resouant scattering off the fast mode.

*S.C. Chili, Phys. Fluids B 3 (1991) 1374. 
A generalized electromagnetic fluctuation dissipation theorem is derived from a test particle approach for arbitrary particle distribution. The general line shape near a resonant pole is calculated to be a Lorentzian shape. The density fluctuations in LHRF at the slow wave (SW) and fast wave (FW) resonance are calculated. It is shown that the resonant fluctuation amplitudes for the slow and fast mode are comparable; however, the contributions from electrons and thermal ions are smaller for the fast wave.

Consider a beam of microwave at frequency $\omega_{\mathrm{I}}$ and wave vector $\vec{k}_{\mathrm{I}}$, incident on a plasma. The plasma particles fluctuate at $\omega$ and $\vec{k}$ and thus generate a scattered wave at $\omega_{\mathrm{s}}, \vec{k}_{\mathrm{s}}$ such that $\omega=\omega_{\mathrm{s}}-\omega_{\mathrm{I}}$ and $\vec{k}=\vec{k}_{\mathrm{s}}-\vec{k}_{\mathrm{I}}$. The differential cross-section per unit frequency $\omega$ and solid angle $\Omega$ is directly proportional to $S(\vec{k}, \omega)$, the spectral function of density correlation defined as

$$
S(\vec{k}, \omega)=\frac{\left\langle\left|\delta n_{\mathrm{e}}\right|^{2}\right\rangle_{\vec{k}, \omega}}{e^{2} n_{0}},
$$

$\delta n_{\mathrm{e}}(\vec{k}, \omega)$ being the fluctuation of charge density at $\vec{k}, \omega$. We concentrate our attention on the investigation of $S(\vec{k}, \omega)$.

A generalized electromagnetic fluctuation-dissipation theorem can be derived for a non-equilibrium plasma of arbitrary distribution using a test particle approach of Ref. 10. In particular,

$$
S(\vec{k}, \omega)=S_{\mathbf{e}}(\vec{k}, \omega)+\sum_{\text {ions }} S_{\mathbf{i}}(\vec{k}, \omega),
$$

where the subscripts "e" and " $\mathrm{i}$ " denote contribution from electrons and ions. It can be shown that the line shape of the scattering function $S(\vec{k}, \omega)$ is generally Lorentzian about a normal plasma mode.

Using Maxwellian distributions for electrons and bulk ions, and a slowing down distribution for $\alpha$-particles, we find that at resonance, $S$ can be expressed in the form

$$
S(\vec{k}, \omega) \simeq \frac{\left|\hat{k} \cdot \overleftrightarrow{\kappa}_{\mathrm{e}} \cdot \hat{e}_{1}^{(0)}\right|^{2}}{\left|\mathcal{K}_{\mathrm{A}}\right|^{2}}\left(\Gamma_{\mathrm{e}}+\sum_{\mathrm{i}} \Gamma_{\mathrm{i}}+\Gamma_{\alpha}\right),
$$

where all the factors on the right hand side can be analytically evaluated for FW and SW. Without going into details of Eq. (3), we mention that the expressions in $S(\vec{k}, \omega)$ are very similar in explicit appearance for the SW and FW. The electron contributions are modified by the factor 


$$
\begin{array}{ll}
\eta_{\mathrm{F}} \equiv \frac{1}{4}\left(\frac{\omega_{\mathrm{pe}}^{2} v_{\mathrm{e}}^{2}}{\Omega_{\mathrm{e}}^{2} c^{2}}\right)^{2}+\left(\frac{\omega_{\mathrm{pe}}}{\Omega_{\mathrm{e}}}\right)^{4} \frac{1}{\left(n_{11}^{2}-S\right)^{2}} & \text { for } \mathrm{FW}, \\
\eta_{\mathrm{s}}=1 & \text { for } \mathrm{SW},
\end{array}
$$

where, here, $S$ is the cold $(1,1)$ diclectric constant. In tokamak parameters, there exists a wide region of parameter space over which $\eta_{\mathrm{F}}$ is small, so that the electron contribution is small for FW.

For bulk ions and $\alpha$ 's, the expressions for FW and SW are almost identical. However, the accessibility condition $\left(n_{\perp}^{2}>0\right)$ of SW and FW implies that the FW has a larger parameter space in $\omega$ and $n_{\|}$over which the wave propagates and where electron damping is weak. Moreover, $S_{\mathrm{i}}$ and $S_{\alpha}$ contain the wave particle resonance factors. For unmagnetized ions, the factors are proportional to $f(\omega / k)$ where $f\left(v_{1}\right)$ is the one-dimensional reduced distribution along the direction of the wave vector $\vec{k}$. Typically $\omega / k_{\perp} F W \sim O\left(v_{A}\right)$, the Alfvén speed, so that $F W$ interacts strongly with energetic ions only. On the other hand, $\omega / k_{\perp}$ sw is often of the order of bulk ion thermal speed and SW is strongly absorbed by bulk ions.

Thus, $S_{\mathrm{i}}(\mathrm{FW}) \ll S_{\mathrm{i}}(\mathrm{SW})$, but $S_{\alpha}(\mathrm{FW}) \sim S_{\alpha}(\mathrm{SW})$. Hence, the scattering function $S(\vec{k}, \omega)$ is of similar magnitude for FW and SW whenever $S_{\alpha}$ dominates, with FW having a larger parameter space satisfying such a condition. This apparently surprising result can be explained by the fact that the dominant component of electric field for both FW and SW are along $\vec{k}_{\perp}$, and when $k_{\perp} \gg k_{11}$, both modes are dominantly electrostatic. The difference is that SW can be approximated by an electrostatic approximation, but FW is intrinsically electromagnetic with a non-trivial, although small, transverse component. As an example, consider an International Thermonuclear Experimental Reactor (ITER)-like parameter: $n_{e}=1 . \times 10^{14} \mathrm{~cm}^{-3}, n_{\mathrm{D}}=n_{\mathrm{T}}$, $T_{e}=T_{\mathrm{D}}=T_{\mathrm{T}}=30 \mathrm{keV}, B_{\mathrm{T}}=4.5 \mathrm{~T}$; taking $\omega=0.8 \omega_{\mathrm{LH}}=5.55 \times 10^{9} \mathrm{sec}^{-1}$, Table I shows the values of $\Gamma_{e},\left(\Gamma_{D}+\Gamma_{c}\right)$, and $\Gamma_{\alpha}$ for $\mathrm{FW}$ for various values of $n_{\|}$ranging from 0 to 3. As can be seen from the table, the $\alpha$-contribution dominates within the range $n_{\|} \leqslant 2.5$. At $n_{\|} \geqslant 3, \omega / k_{\mathrm{F}} \geqslant v_{\alpha}$ and there is no contribution from $\alpha$ 's in the unmagnetized approximation. Generally, there exists a wide range of $\omega$ over which there exists some range of $n_{\|}$in which $\Gamma_{\alpha}$ will dominate. 
TABLE I

Comparison BetweEn $\alpha$ AND BUlK Contributions FOR AN ITER-LIKE PLASMA

\begin{tabular}{|c|c|c|c|c|c|}
\hline \hline$n_{11}$ & 0 & 1 & 1.5 & 2.5 & 3. \\
\hline$k_{\mathrm{F}}\left(\mathrm{cm}^{-1}\right)$ & 14.52 & 10.66 & 7.86 & 5.06 & 4.27 \\
\hline$\frac{\omega}{k_{\mathrm{F}} v_{\alpha}}$ & 0.294 & 0.401 & 0.544 & 0.845 & 1. \\
\hline$v_{\mathrm{c}} g_{0 \alpha}^{(1)}$ & 0.405 & 0.329 & 0.227 & 0.059 & 0 \\
\hline$\eta_{\mathfrak{f}}$ & - & - & $2.76 \times 10^{-2}$ & $5.98 \times 10^{-3}$ & $3.53 \times 10^{-3}$ \\
\hline$\omega \Gamma_{\alpha}(\mathrm{FW})$ & 0.128 & 0.142 & 0.133 & 0.054 & 0 \\
\hline$\omega \Gamma_{\mathrm{e}}(\mathrm{FW})$ & 0 & 0 & $4.27 \times 10^{-3}$ & $6.33 \times 10^{-3}$ & $4.72 \times 10^{-3}$ \\
\hline$\omega\left(\Gamma_{\mathrm{D}}+\Gamma_{\mathrm{T}}\right)$ & $2.73 \times 10^{-2}$ & $4.45 \times 10^{-4}$ & $2.15 \times 10^{-7}$ & $7 \times 10^{-18}$ & $4 . \times 10^{-25}$ \\
\hline \hline
\end{tabular}

We, thus, conclude that from the point of view of $\alpha$-diagnostics by microwave scattering, the electromagnetic calculations give a more optimistic (and more realistic) picture in that there exists appreciable range of parameters in which $\alpha$-contributions can dominate in resonance scattering. Because of the ordering $\omega_{\mathrm{pe}}^{2} / \omega^{2} \sim O\left(10^{3}\right) \gg 1$, the resonant scattering amplitude is often two to three orders of magnitude larger than off-resonance amplitudes. One thus has a larger signal to noise ratio near resonance which, in turn, implies a lower microwave power required. Detailed numerical computation is in progress.

This is a report of work sponsored by the U.S. Department of Energy under Contract No. DE-AC03-89ER53277.

\section{References}

[1] A.I. Akhiezer et al., Collective Oscillations in a Plasma (English trans. by H.S.S. Massey) (The M.I.T. Press, Cambridge, 1967).

[2] G. Bekefi, Radiation Processes in Plasmas (John Wiley \& Sons, Inc., New York, 1966).

[3] J. Sheffield, Plasma Scattering of Electromagnetic Radiation (Academic Press, New York, 1975).

[4] C.M. Surko and R.E. Slusher, Phys. Fluids 23 (1980) 2425. 
[5] D.P. Hutchinson et al., Rev. Sci. Instrum. 56 (1985) 1075.

[6] L. Vahala, G. Vahala, D.J. Sigmar, Nucl. Fusion 26 (1986) 51.

[7] L. Vahala G. Vahala, D.J. Sigmar, Nucl. Fusion 28 (1988) 1595.

[8] T.P. Hughes and S.R.P. Smith, Nucl. Fusion 28 (1988) 1451.

[9] K.L. Wong, Bull. Am. Phys. Soc. 34 (1989) 2014.

[10] M.N. Rosenbluth and N. Rostoker, Phys. Fluids 5 (1962) 776. 


\subsubsection{FY'91 PUBLICATIONS}

G.G. Castle (UTA), S.C. McCool (UTA), M.E. Austin (Auburn U), D.L. Brower (UCLA), R.K. Fisher, G.A. Hallock (UTA), and P.B. Parks, Impurity Pellet Ablation in TEXT (Abstract), Bull. Am. Phys. Soc. 35 (1990) 2028.

V.S. Chan and S.C. Chiu, Kinetic Aspects of Nonresonant Forces by RF Waves (Abstract), Bull. Am. Phys. Soc. 36 (1991) 2339.

S.C. Chiu, T.K. Mau (UCLA), and R.W. Harvey, Theoretical Modeling of Fast Wave Current Drive in DIII-D (Abstract), Bull. Am. Phys. Soc. 36 (1991) 2470.

S.C. Chiu, On the Collective Scattering of Microwaves by Fusion Plasmas, GA-A20421 (1991).

S.C. Chiu, Influence of Fast Waves on the Collective Scattering of Microwaves in Fusion Plasmas, GA-A20539 (1991), to be published in Physica Scripta.

M.S. Chu and R.R. Dominguez, Effect of Finite $\epsilon \beta_{\mathrm{p}}$ on the Ion Temperature Gradient Mode in a Tokamak (GA-A20002), Nucl. Fusion 31 (1991) 951.

M.S. Chu, L.L. Lao, A.D. Turnbull, and J.K. Lee, Toroidal Effect and the Damping of MHD Modes in CART (Abstract), Bull. Am. Phys. Soc. 36 (1991) 2309.

R.R. Dominguez and G.M. Staebler, Impurity Induced Thermal Pinches and Anomalous Impurity Transport (Abstract), Bull. Am. Phys. Soc. 36 (1991) 2344.

R.R. Dominguez, Isotope Scaling in the Drift Wave Model, GA-A20184 (1991), submitted to Nuclear Fusion.

R.R. Dominguez, DIII-D Hot Ion Plasma Simulations, GA-A20382 (1991), to be published in Nuclear Fusion.

R.K. Fisher, J.M. McChesney, A.M. Howald, P.B. Parks, D.M. Thomas, S.C. McCool (UTA), and W.L. Rowan (UTA), Fast Alpha Diagnostics Using Carbon Pellet Injection (GA-A20107), Rev. Sci. Instrum. 61 (1990) 3196.

R.K. Fisher, J.M. McChesney, P.B. Parks, J.A. Snipes (MIT), J.L. Terry (MIT), E.S. Marmar (MIT), S.J. Zweben (PPPL), and S.S. Medley (PPPL), Impurity Pellet Alpha Diagnostic Experiments on TFTR (Abstract), Bull. Am. Phys. Soc. 36 (1991) 2452.

R.K. Fisher, J.M. McChesney, P.B. Parks, J.L. Terry (MIT), E.S. Marmar (MIT), D.W. Johnson (PPPL), and S.S. Medley (PPPL), Initial Fast Alpha Diagnostic Pellet Injection Experiments on TFTR (Abstract), Bull. Am. Phys. Soc. 35 (1990) 2091. 
R.W. Harvey, M.R. O'Brien (Culham Laboratory), V. Rozhdestvenskiy (Ioffe Institute), T.C. Luce, M.G. McCoy (NERSC), and G.D. Kerbel (NERSC), Electron Cyclotron Emission from Nonthermal Tokamak Plasmas, GA-A19642 (1990), submitted to Physics of Fluids B.

R.W. Harvey and R.O. Dendy (UKAEA/Culham Laboratory), A Trapped-Passing Fluid Model for Tokamak Neoclassical Transport, GA-A20029 (1991), submitted to Physics of Fluids B.

R.W. Harvey, S.C. Chiu, M.G. McCoy (NERSC), G.D. Smith (NERSC), and T.K. Mau (UCLA) 3D Fokker-Planck Calculation of Fast Wave and Electron $\mathrm{Cy}$ clotron Current Drive in a Tokamak Reactor (Abstract), Bull. Am. Phys. Soc. 36 (1991) 2340.

F.J. Helton and J.M. Greene, $A$ Numerical Technique for Obtaining the Complete Bifurcated Equilibria Solution Space for a Tokamak (GA-A19624), to be published in Journal of Computational Physics.

F.J. Helton, J.M. Greene, and J.W. Helton (UCSD), A Measure of Control Sensitivity and Saturation Effects for Tokamak Equilibria (GA-A19323), Plasma Phys. and Contr. Fusion 33 (1991) 827.

F.J. Helton, J.M. Greene, and T.S. Taylor, Design of Feedback Control Systems (Abstract), Bull. Am. Phys. Soc. 36 (1991) 2478.

F.J. Helton, J.M. Greene, T. Ohkawa, P.A. Politzer, and A.D. Turnbull, Ideal MHD Properties of an Ellipsoidal Shell Tokamak (GA-A18443), Nucl. Fusion 31 (1991) 487.

F.L. Hinton, Thermal Confinement Bifurcation and the $L$ - to $H$-mode Transition in Tokamaks (GA-A20151), Phys. Fluids B 3 (1991) 696.

F.L. Hinton and J.C. Wiley (UT), Particle and Energy Confinement Bifurcation in Tokamaks (Abstract), Bull. Am. Phys. Soc. 36 (1991) 2475.

T.H. Jensen, J.M. Greene, and P.A. Politzer, Large Anomaly of the Perpendicular Resistivity in a Tokamak GA-A20557 (1991), to be published in Physics of Fluids B.

Y.B. Kim (UCSD) and F.L. Hinton, Poloidal Viscosity and Radial Currents (University of California at San Diego Preprint, 1991), to be published in Physics of Fluids B.

Y.R. Lin-liu and V.S. Chan:

Characteristic Impedance of a Soliton-Bearing Nonlinear Transmission Line, GA-A20378 (1991), Microwave and Optical Technology Letters 4 (1991) 468. 
O.T. Kingsbury (UCSD) and R.E. Waltz, 2D-Numerical Simulation of Trapped Ion Turbulence in a Tokamak (Abstract), Bull. Am. Phys. Soc. 36 (1991) 2279.

O.T. Kingsbury (UCSD) and R.E. Waltz, 2D-Numerical Simulation of Trapped Electton and Trapped Ion Mode Turbulent Transport in a Tokamak (Abstract), Bull. Am. Phys. Soc. 35 (1990) 1980.

O.T. Kingsbury (UCSD) and R.E. Waltz, 2-D Numerical Simulation of Trapped Electron Mode Turbulent Transport in a Tokamak, GA-A20381 (1991), to be published in Physics of Fluids B.

R.L. Miller, V.S. Chan, and T. Ohkawa, Minimum Energy Toroidal Pinch Equilibria with Finite Pressure (Abstract), Bull. Am. Phys soc. 36 (1991) 2308.

P.B. Parks, Pellet Ablation Flow Near the Stagnation Plane at Low Magnetic Reynolds Number (GA-A20274), Nucl. Fusion 31 (1991) 1431.

G.M. Staebler and R.R. Dominguez, Drift Wave Transport Reduction by Electric Field Profile Control (Abstract), Bull. Am. Phys. Soc. 36 (1991) 2344.

G.M. Staebler and R.R. Dominguez, Electric Field Effects on Ion Temperature Gradient Modes in a Sheared Slab, GA-A20406 (1991), Nucl. Fusion 31 (1991) 1891.

A.D. Turnbull, The Toroidal Kink Mode at Finite Beta, GA-A20270 (1991), to be published in Nuclear Fusion.

A.D. Turnbull, P.A. Politzer, M.S. Chu, C.D. Challis (JET), G.D. Porter (LLNL), L.L. Lao, and E.J. Strait, Stability of High $\epsilon \beta_{\mathrm{p}}$ Discharges in DIII-D (Abstract), Bull. Am. Phys. Soc. 35 (1990) 1972.

A.D. Turnbull, M.S. Chu, J.M. Greene, M.S. Chance, E.J. Strait, W.W. Heidbrink (UCI), and T.S. Taylor, The Shear Alfvén Spectrum in Tokamak Geometry (Abstract), Bull. Am. Phÿs. Soc. 36 (1991) 2377.

R.E. Waltz, G.D. Kerbel (NERSC), G.W. Hammett (PPPL), and R.R. Dominguez, Simulation of Turbulence with Gyro-Landau Fluid Models (Abstract), Bull. Am. Phys. Soc. 36 (1991) 2279.

R.E. Waltz, J.C. DeBoo, and M.N. Rosenbluth (UCSD), Magnetic Field Scaling of Dimensionally Similar Tokamak Discharges (GA-A20172), Phys. Rev. Lett. 65 (1990) 2390.

R.E. Waltz, J.C. DeBoo, M.N. Rosenbluth (UCSD), C.W. Barnes (LANL), R.J. Goldston (PPPL), D.W. Johnson (PPPL), F.W. Perkins (PPPL), S.D. Scott (PPPL), and M.C. Zarnstorff (PPPL), Tokamak Ignition Projections from 
APP REPORT TO DOE, FY 89 THROUGH FY91

Dimensionally Similar Discharges (GA-A20267), in Plasma Physics and Controlled Nuclear Fusion Research (Proc. 13th Int. Conf. Washington, 1990), IAEA, Vienna, Vol. 2, p. 273. 


\subsection{HIGHLIGHTS OF FY90}

Technical progress has been made on a variety of topics. Synopses of these studies are summarized in our quarterly GA Theory News. A majority of the work has been submitted for publication in refereed journals as listed in the publications section and/or presented at various conferences. Three exemplary summary articles are included in this section.

One of the most urgent problems in current fusion research is to understand the underlying mechanism(s) responsible for the $\mathrm{H}$-mode in tokamaks. Recent results from DIII-D [2-5] have shown that edge poloidal rotation increases dramatically during the $\mathrm{L}-\mathrm{H}$ transition, and remains large in $\mathrm{H}$-mode. This had led to several suggestions [2-6,7] about how poloidal rotation can trigger improved confinement. Of particular interest here is the result of Biglari et al. [2-7] that edge turbulence can be significantly reduced by sheared poloidal effects. Hinton [2-8] has formulated a simple model for the suppression of anomalous transport by velocity shear, joined with standard results on neoclassical poloidal rotation. It is shown that these elements are sufficient to obtain a bifurcation in the energy confinement, reminiscent of the $\mathrm{L}-\mathrm{H}$ transition. The predicted temperature profiles develop an edge pedestal (increasing the edge poloidal rotation) when the heating power exceeds a threshold value. The model predicts critical values of density and magnetic field, which must be exceeded to achieve bifurcation. In addition, the scaling of the power threshold with density and magnetic field is similar to experimental results.

Another pressing issue in the confinement area is a better understanding of bulk plasma energy confinement. Since tokamaks can operate in a variety of different confinement regimes, one would speculate that there are several bulk transport mechanisms possible (likely acting simultaneously). Furthermore, the underlying physics in the bulk and edge plasma regions is also likely to be different. Thus, even if it were possible to better understand each transport mechanism separately, a realistic, quan-. titatively accurate global transport model, in which all of the constituent elements are strongly interacting, appears to be unfeasible. However, Waltz et al. [2-9] have pointed out that this lack of complete information does not necessarily prevent one from being able to formulate an accurate scaling of present tokamaks into the ignition regime, where the devices will have a larger size $(a)$ and a stronger magnetic field $(B)$. This is possible because the most general dimensional constraints on diffusive transport mechanisms [2-10] allow one to deduce a very simple scaling of energy confinement with $a$ and $B$ in "dimensionally similar" discharges [2-9]. Waltz et al. [2-9] note that there are only two possibilities for this scaling in dimensionally similar discharges: either Bohm-like, $\tau_{\mathrm{E}} \propto B^{1 / 3} a^{5.3}$ or gyroBohm-like, $\tau_{\mathrm{E}} \propto B^{5 / 2}$. DIII-D 
L-mode experiments motivated by this work have concluded that gyroBohm scaling is the actual diffusive process. Accordingly, confinement experiments accrue significant benefit from both larger $a$ and $B$ since the ignition parameter $N T \tau_{\mathrm{E}} \propto B^{3} A^{5 / 2}$; note that for Bohm processes $N T \tau_{\mathbb{E}} \propto B^{7 / 3} a^{5 / 3}$.

A third area where important theoretical contributions have been made is in steady-state current drive in plasmas using wave helicity injection. This method exploits the fact, first note for circularly polarized waves [2-11], that wave helicity $\langle\underline{A} \cdot \underline{B}\rangle$ ( $\underline{A}$ is the magnetic vector potential, $\underline{B}$ the associated magnetic field, and \langle\rangle denotes a time average (can be transferred to the plasma, driving current in the plasma. Chan et al. [2-12] generalized this concept to waves of arbitrary polarization and damping direction, and also developed a simple physical picture to describe the various effects contributing to plasma current generation. An important practical application to tokamaks is that the current drive efficiency is independent of density, while strongly dependent on electron temperature. Thus, in a high density reactor relevant temperature regime this method becomes more efficient than other wave current drive mechanisms.

During FY90, our theorists have made extended visits to ASDEX, T-10, and $\mathrm{JT}-60$ through the international collaboration program. In return, over twenty scientists from other institutions have visited the GA theory group. We organized a DOE workshop on IBW Heating and a US/Japan Workshop on Wave Helicity Injection and Current Drive. Our collaboration with UCSD plasma theorists continued to produce a number of important publications. 


\subsection{HIGHLIGHTS OF FY89}

One of the major strengths of the MHD program is the close interaction 'batween theory and experiment. In the past fiscal year, this close collaboration has produced major understandings of plasma profile effects on $\beta$ limits [2-13], the role of the internal mode versus the external mode and ideal mode versus resistive mode in high $\beta$ plasmas [2-14], and the relationship between ballooning modes and edge localized modes (ELMs). As a result, DIII-D can now consistently operate at reactor-rele rant $\beta$ values by avoiding the most virulent modes, which may be responsible for major disruptions, through plasma shaping and profile control.

Much of the progress in the transport area has followed from the clarification of the experimental situation. For example, with the very flat density profile observed in $\mathrm{H}$-mode plasmas, previous theoretical prediction of $\eta_{1}$-mode threshold has to be significantly modified. It no longer depends on $\eta_{1}$, but on the ion temperature gradient [2-15]. Similar modification has to be made for the DIII-D hot ion H-mode regime when the ion temperature is much higher than the electron temperature. Our understanding of the edge transport in a diverted plasma has been expanding. Predictions of particle and heat fluxes are possible with existing. It has contributed heavily in the planning of the Advanced Divertor Experiment on DIII-D.

Electron cyclotron heating is an important element of the DIII-D experimental program. Theory routinely supports this experiment by predicting and analyzing key physics quantities such as absorption profile, energetic electron distribution, and rf-driven current. Fast wave heating near the ion cyclotron frequency offers proven technique with readily available technology. Theory has provided a broad range of support for this experiment. Fast wave coupling to H-mode plasma is reasonably wellunderstood. Theory also predicts good current drive efficiency for fast wave provided the target plasma reaches a sufficiently high electron temperature [2-16]. Fast wave current drive is being planned for DIII-D. 


\subsection{REFERENCES FOR SECTION 2}

[2-1] A. Pletzer and R.L. Dewar, to be published in Journal of Plasma Physics.

[2-2] M.S. Chu, J.M. Greene, M. Klasky, and M.S. Chance, General Atomics Report GA-A20135 (1990), submitted to Journal of Computational Physics.

[2-3] R.R. Dominguez, General Atomics Report GA-A20184, to be published in Nuclear Fusion.

[2-4] S.C. Chiu, General Atomics Report GA-A20176, Phys. Fluids B 3 (1991) 1374.

[2-5] R.J. Groebner, K.H. Burrell, and R.P. Seraydarian, Phys. Rev. Lett. 64 (1990) 3015.

[2-6] K.C. Shiang and E.C. Crume, Jr., Phys. Rev. Lett. 63 (1989) 2369.

[2-7] H. Biglari, P.H. Diamond, and P.W. Terry, Phys. Fluids B 2 (1990) 1.

[2-8] F.L. Hinton, Phys. Fluids B 3 (1991) 696.

[2-9] R.E. Waltz, J.C. DeBoo, and M.N. Rosenbluth, Phys. Rev. Lett. 65 (1991) 2390.

[2-10] J.W. Connor and J.B. Taylor, Nucl. Fusion 19 (1977) 1047.

[2-11] T. Ohkawa, Comments on Plasma Physics and Controlled Fusion 12 (1989) 165.

[2-12] V.S. Chan, R.L. Miller, and T. Ohkawa, Phys. Fluids B 2 (1990) 944 and 1441.

[2-13] A.D. Turnbull, M.S. Chu, J.M. Greene, and F.J. Helton, General Atomics Report GA-A19524 (1989), submitted to Nuclear Fusion.

[2-14] J.K. Lee, E.J. Strait, L.L. Lao, and T.S. Taylor, Nucl. Fusion 29 (1989) 1181.

[2-15] R.R. Dominguez and R.E. Waltz, Phys. Fluids 31 (1988) 3147.

[2-16] S.C. Chiu, V.S. Chan, R.W. Harvey, and M. Porkolab, Nucl. Fusion 29 (1989) 2175. 


\section{ALPHA PARTICLE DIAGNOSTIC}

\subsection{FY91 HIGHLIGHTS}

Two major developments occurred in FY91. First, the favorable wall conditioning results observed during lithium pellet injection into TFTR [3-1] resulted in our examining the use of lithium pellet ablation clouds as a target for alpha diagnostics. The surprising result was an increase in signal level of order $10^{3}$ over that expected for carbon pellets. This very favorable result was presented at the "First Workshop on Alpha Physics in TFTR" held at PPPL in March 1991.

Second, as a result of this workshop and a second workshop held in April 1991 at PPPL to specifically discuss impurity pellet injection experiments during D-T operation, we began a collaboration with PPPL and MIT scientists to observe helium neutrals from alphas interacting with lithium pellet clouds during ICH minority heating experiments on TFTR.

\subsubsection{USE OF LITHIUM PELLET CLOUD TARGET}

The ablation cloud surrounding an injected lithium pellet should have a large spatial region dominated by singly-ionized $\mathrm{Li}^{+}$ions, just as a carbon pellet cloud should contain a large $\mathrm{C}^{4+}$ region. The ions prefer to hang on to their last two electrons.

Figure 3-1 shows $\mathrm{Li}^{+}$light at $5485 \AA$ from a $1.5 \mathrm{~mm}$ long by $2 \mathrm{~mm}$ diameter cylindrical lithium pellet injected into TFTR at $\sim 750 \mathrm{~m} / \mathrm{sec}$. The $\mathrm{Li}$ length extends approximately 20 to $30 \mathrm{~cm}$ from the pellet in each direction along the magnetic field. The local $T_{\mathrm{e}} \sim 2.3 \mathrm{keV}$ and $n_{\mathrm{e}} \sim 7 \cdot 10^{13} \mathrm{~cm}^{-3}$ for this shot (TFTR \#52197). The next step is to measure the size of $\mathrm{Li}^{0}$ and $\mathrm{Li}^{2+}$ clouds undez comparable plasma conditions to check the assumption that there is a large region of the cloud dominated by one $\mathrm{Li}^{+}$ionization state.

Alpha particles passing through the $\mathrm{Li}^{+}$portion of ine cloud will interact via double charge exchange interactions 


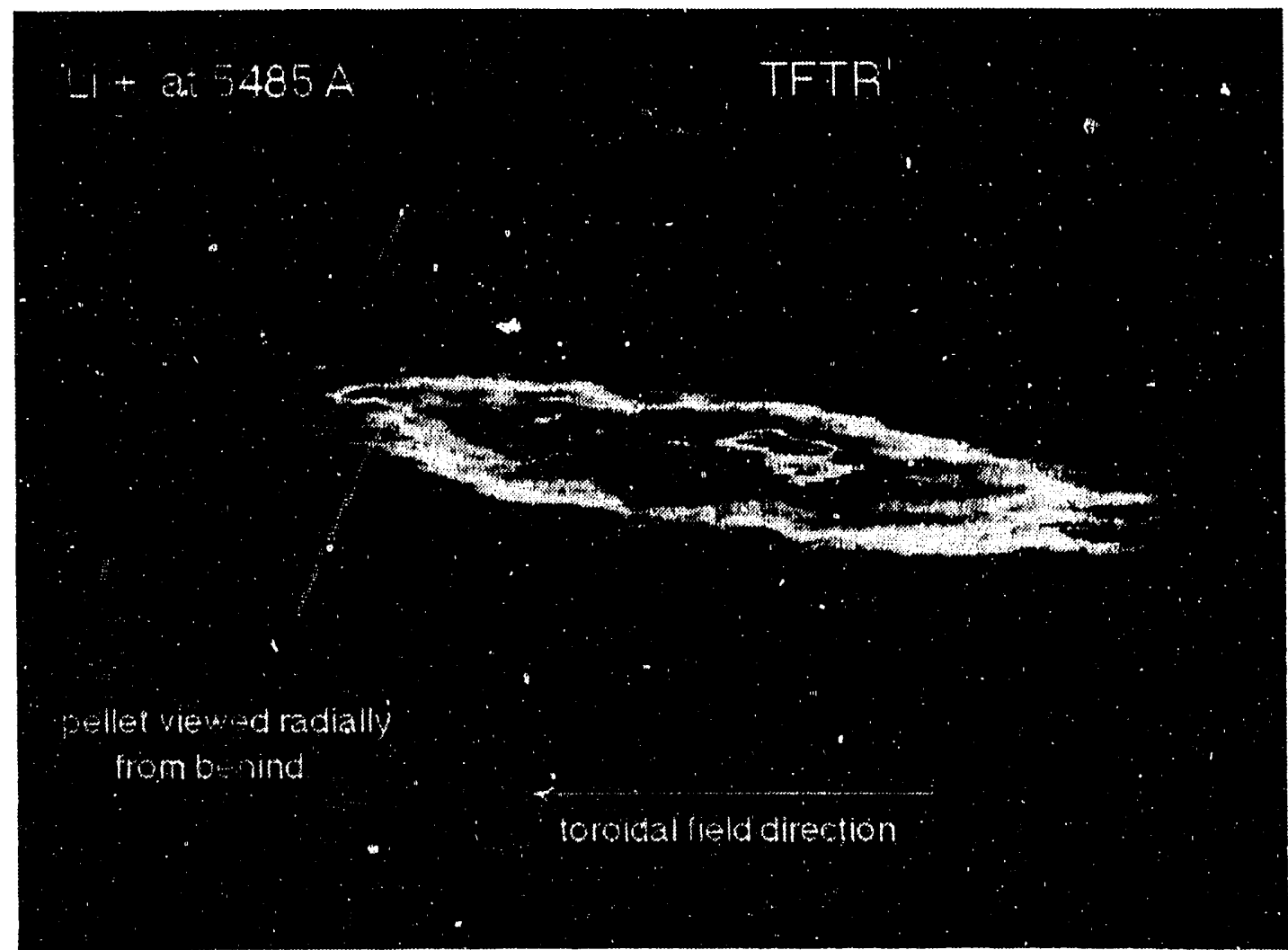

FIG. 3-1. $\mathrm{Li}^{+}$light at $5485 \AA$ from a lithium pellet injected into TFTR. The light extends 20 to $30 \mathrm{~cm}$ from the pellet in each direction along the magnetic field.

$$
\mathrm{He}^{2+} \mathrm{Li}^{+} \longrightarrow \mathrm{He}^{0}+\mathrm{Li}^{3+},
$$

and sequential single charge exchange interactions

$$
\mathrm{He}^{2+}+\mathrm{Li}^{+} \longrightarrow \mathrm{He}^{0}+\mathrm{Li}^{2+},
$$

followed by

$$
\mathrm{He}^{+}+\mathrm{Li}^{+} \longrightarrow \mathrm{He}^{0}+\mathrm{Li}^{2+} \text {. }
$$


The expected line integral cloud density on TFTR is sufficient to create an equilibrium fraction of helium neutrals via single and double charge exchange interactions. At incident alpha energy $\sim 1 \mathrm{MeV}$, sequential single capture interactions become the dominant source of neutrals.

By measuring the energy distribution of neutral helium atoms $d n_{\mathrm{He}^{\circ}} / d E$ created in the region of the cloud dominated by $\mathrm{Li}^{+}$ions, we can determine the incident fast confined alpha energy distribution

$$
\frac{d n_{\mathrm{He}^{2}+}}{d E}=\frac{d n_{\mathrm{He}^{0}}}{d E} \div F_{0}^{\infty}(E, Z=1)
$$

where $F_{0}^{\infty}(E, Z=1)$ is the equilibrium fraction shown in Fig. 3-2 for helium incident on a $\mathrm{Li}^{+}$target. Also shown in Fig. 3-2 is the equilibrium fraction for a $\mathrm{C}^{4+}$ target. The lithium $F_{0}(E)$ is larger due to the increase in the electron capture cross-sections (the $\mathrm{K}$-shell radius is larger for lithium) and a decrease in the cross-section for reionization of the neutrals (the $Z$ for lithium is lower). One very important result of the increased signal level for a $\mathrm{Li}^{+}$target is that single-particle alpha particle confinement can be studied prior to $\mathrm{D}-\mathrm{T}$ operation of TFTR using $3.7 \mathrm{MeV}$ alphas from $\mathrm{D}-\mathrm{He}^{3}$ reactions occurring in today's TFTR ICH $\mathrm{He}^{3}$ minority heating experiments (see Subsection 3.1.3). 


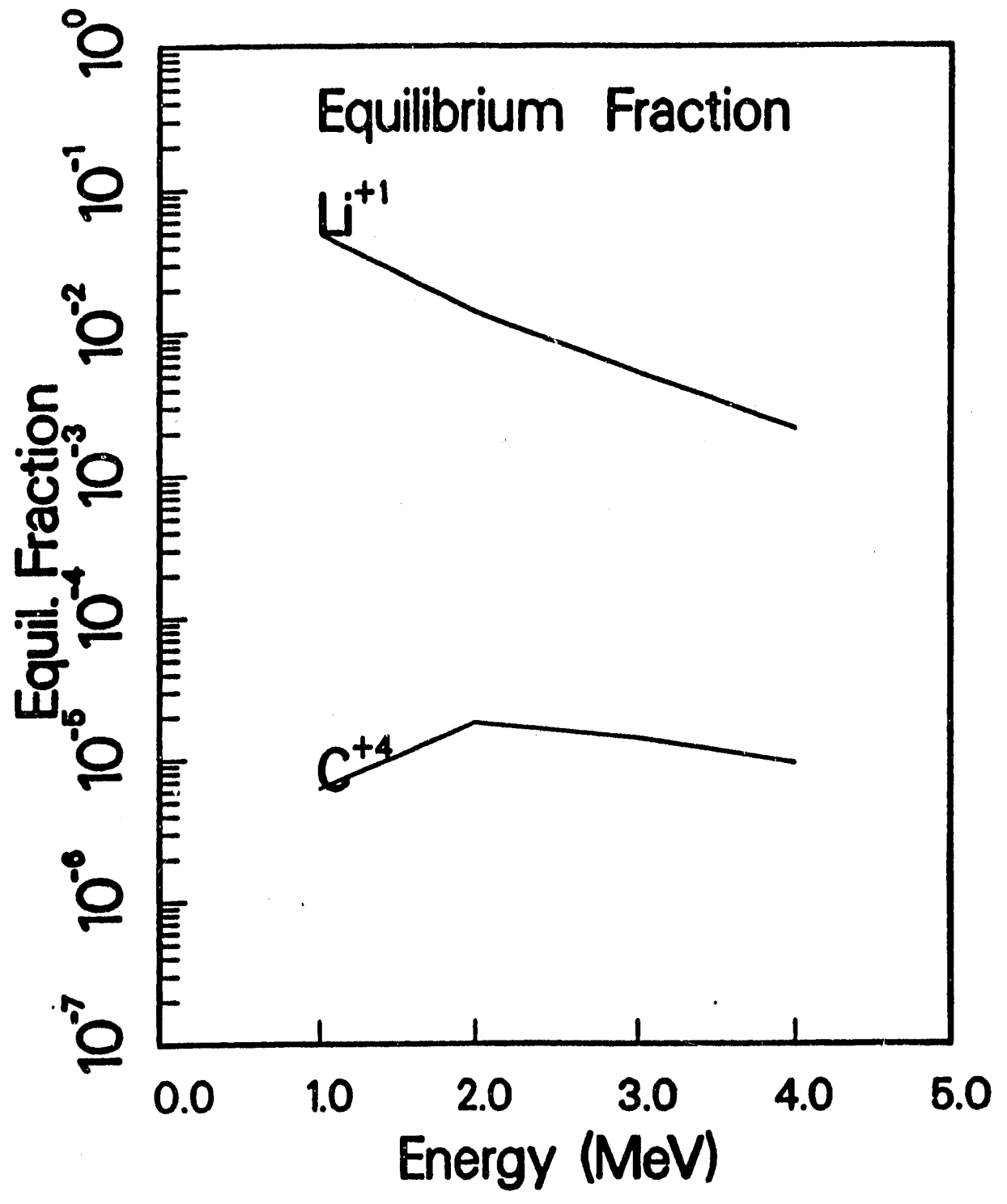

FIG. 3-2. Calculated neutral equilibrium fractions for ${ }^{4} \mathrm{He}$ passing through a $\mathrm{Li}^{+}$target and a $\mathrm{C}^{4+}$ target. 


\subsubsection{FALL 1991 NEUTRAL MEASUREMENTS ON TFTR}

At the April 1991 "Impurity Pellet DT Workshop" held at PPPL, it was agreed that we should attempt to observe charge exchange neutrals from the interaction of ICH heated ${ }^{3} \mathrm{He}$ ions into pellet ablation clouds during fall 1991 TFTR operation. A simple analyzer (shown schematically in Fig. 3-3) has been designed and built. It consists of a thin carbon foil to strip the escaping fast neutrals and a thin $\mathrm{ZnS}$ scintillator to detect the resulting fast ions which are bent downward as shown by the residual toroidal field of the tokamak.

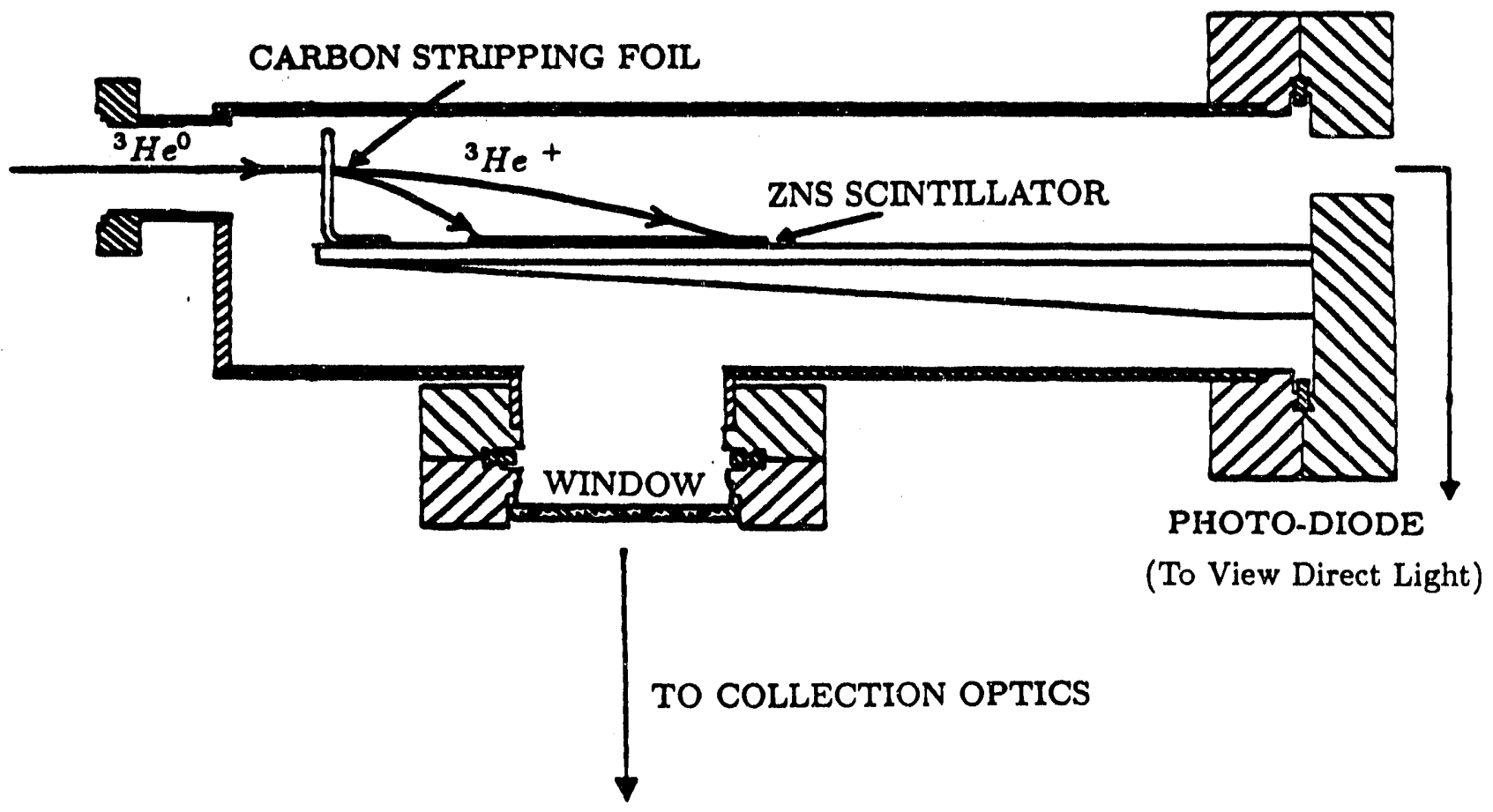

FIr. 3-3. Fall 1991 neutral particle analyzer designed to observe ${ }^{3}$ Fie and D neutrals from TFTR.

The carbon foil thickness is $250 \AA$ which results in approximately $20 \mathrm{keV}$ of energy loss for a $100 \mathrm{keV}$ incident ${ }^{3} \mathrm{He}$. Thinner foils were too fragile to be reliable. The residual TFTR toroidal field varies between 7 and $10 \mathrm{kG}$ over the length of the analyzer which is located between two toroidal field coils. The $\mathrm{ZnS}(\mathrm{Cu})$ scintillator thickness was chosen to be slightly larger than the range of $3.5 \mathrm{MeV}$ alphas to be 
observed in later experiments. It is thin to limit the neutron- and gamma rayinduced background. It is covered by $1500 \AA$ of aluminum to block plasma and pellet background light. If necessary, an interference filter can be used to select scintillation wavelength light and reduce the background light from the pellet. This analyzer will be installed on TFTR in October 191.

\subsubsection{TFTR EXPERIMENTS}

We have begun studying possibilities for a high energy ( 1 to $3.7 \mathrm{MeV}$ ) neutral particle analyzer for 1992 and beyond TFTR experiments. The analyzer would be similar to that shown schematically in Fig. 3-4, where electrical and magnetic deflection is used for energy and mass discrimination. Alternatives under consideration include using an analyzer built by M. Petrov of the Ioffe Institute in St. Petersburg, Russia. This analyzer is being tested at JET in 1991 but we have not been able to obtain information on their results to date.

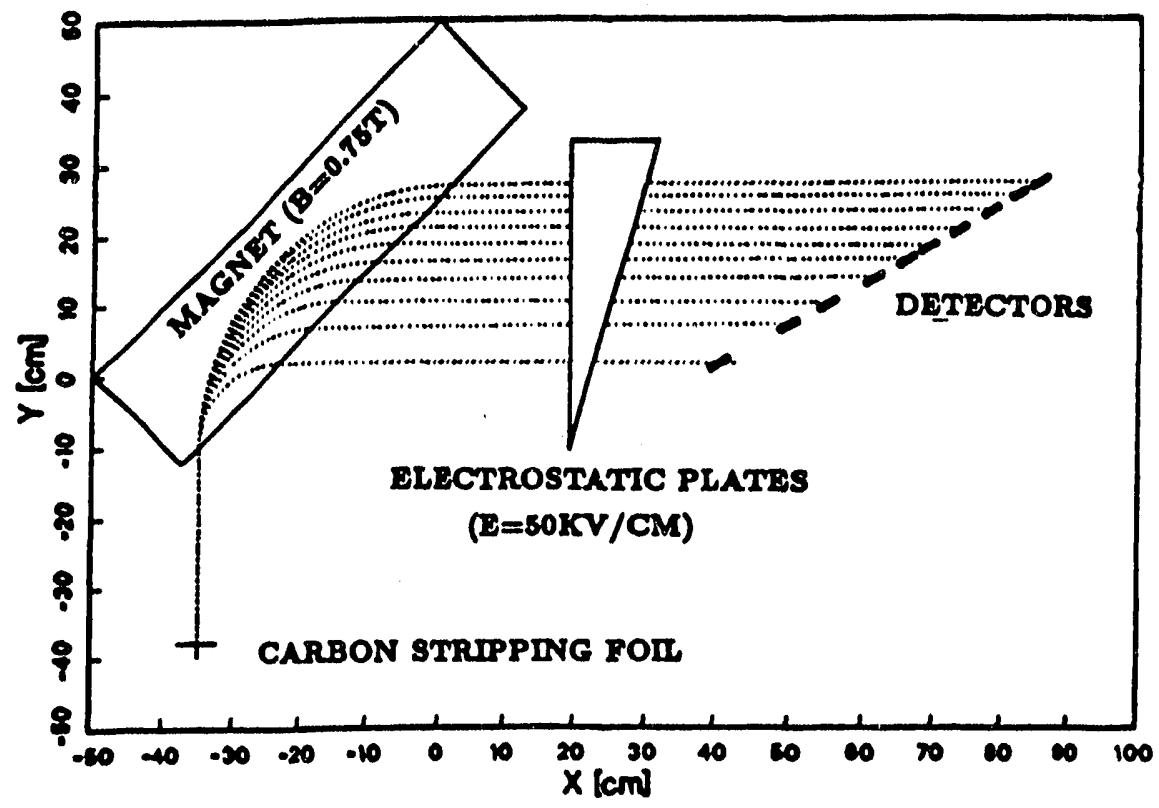

FIG. 3-4. Schematic of high energy analyzer needed for measuring $37 \mathrm{MeV}$ alphas from $\mathrm{D}-{ }^{3} \mathrm{He}$ reactions in 1992 and $3.5 \mathrm{MeV}$ alphas from $\mathrm{D}-\mathrm{T}$ reactions in 1993-94. 
Another possibility suggested by Princeton is to modify an existing TFTR analyzer. This would require decreasing the magnet pole spacing to bend high energy alphas, adding an electric deflection section to separate ${ }^{4} \mathrm{He}$ from ${ }^{3} \mathrm{He}$ and ${ }^{4} \mathrm{He}^{2+}$ from ${ }^{4} \mathrm{He}^{+}$, and installing thin scintillators to replace the existing microchannel plate.

Either of the above options should be less expensive than building a totally new analyzer. They both will require keeping the photomultipliers outside the TFTR but all with long fiberoptic cables to carry the scintillation light to the phototubes.

The goals of the 1992 experiments would be to measure the high energy $(\geqslant 100 \mathrm{keV}){ }^{3} \mathrm{He}$ ion tail created during $\mathrm{ICH}$ minority heating experiments, and to measure the $3.7 \mathrm{MeV}{ }^{4} \mathrm{He}$ 's created by ${ }^{3} \mathrm{He}-\mathrm{D}$ reactions in these same plasmas. Figure 3-5 shows the ${ }^{3} \mathrm{He}$ signal levels for a modified TFTR analyzer installed in the basement of TFTR. Figure 3-6 shows the calculated $3.7 \mathrm{MeV}$ alpha signal for an Ioffe-style analyzer installed in the TFTR basement. Large signal levels are expected as shown.

The same high energy analyzer could also be used for $3.5 \mathrm{MeV}$ alpha measurements during L-T experiments on TFTR. Figure 3-7 also shows the calculated $3.5 \mathrm{mev}$ alpha signal during $\mathrm{D}-\mathrm{T}$ operation for an Ioffe-type analyzer in the TFTR basement.

We also plan to measure the spatial distribution of $\mathrm{Li}^{0}, \mathrm{Li}^{+}$, and $\mathrm{Li}^{2+}$ in the pellet clouds to verify that a suitable could region is dominated by the helium-like $\mathrm{Li}^{+}$charge state. We also hope to study boron pellets since boron is expected to offer better pellet penetration than lithium with a signal level between lithium and carbon.

\subsubsection{CONCLUSIONS}

In summary, lithium pellet injection looks extremely attractive as a fast confined alpha particle diagnostic. We have planned a series of experiments that should result in a fully-tested diagnostic prior to $\mathrm{D}-\mathrm{T}$ operation of TFTR. 


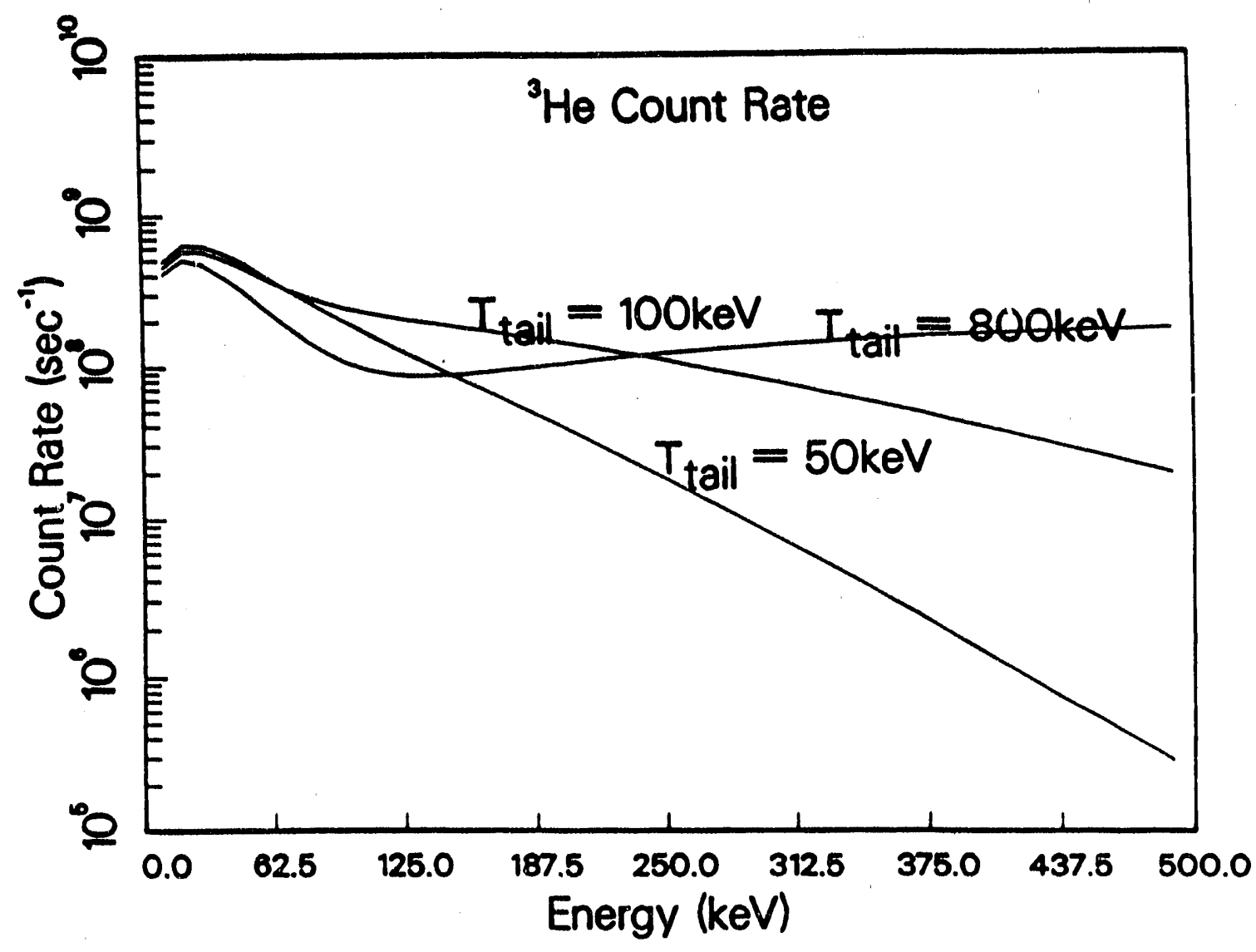

Fig. 3-5. Calculated ${ }^{3} \mathrm{He}$ signal levels from a modified TFTR neutral particle analyzer in basement of TFTR. ${ }^{3} \mathrm{He}$ is due to ICH minority heating in ion tail. 


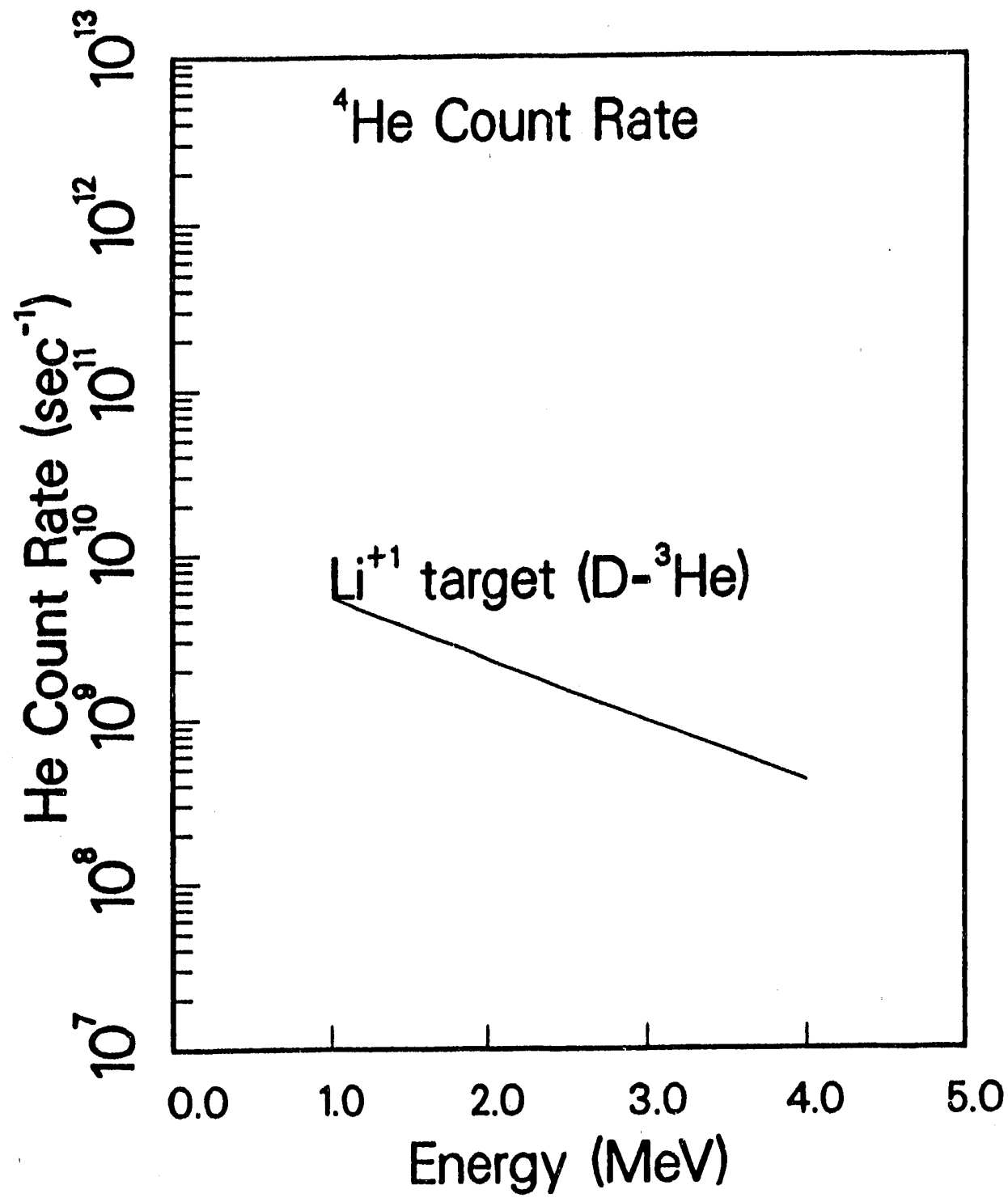

Fig. 3-6. Calculated $3.7 \mathrm{MeV}{ }^{4} \mathrm{He}$ signal from alphas created by ${ }^{3} \mathrm{He}-\mathrm{D}$ reactions in ICH heated TFTR plasmas. Analyzer assumed similar to Ioffe analyzer in basement of TFTR. 


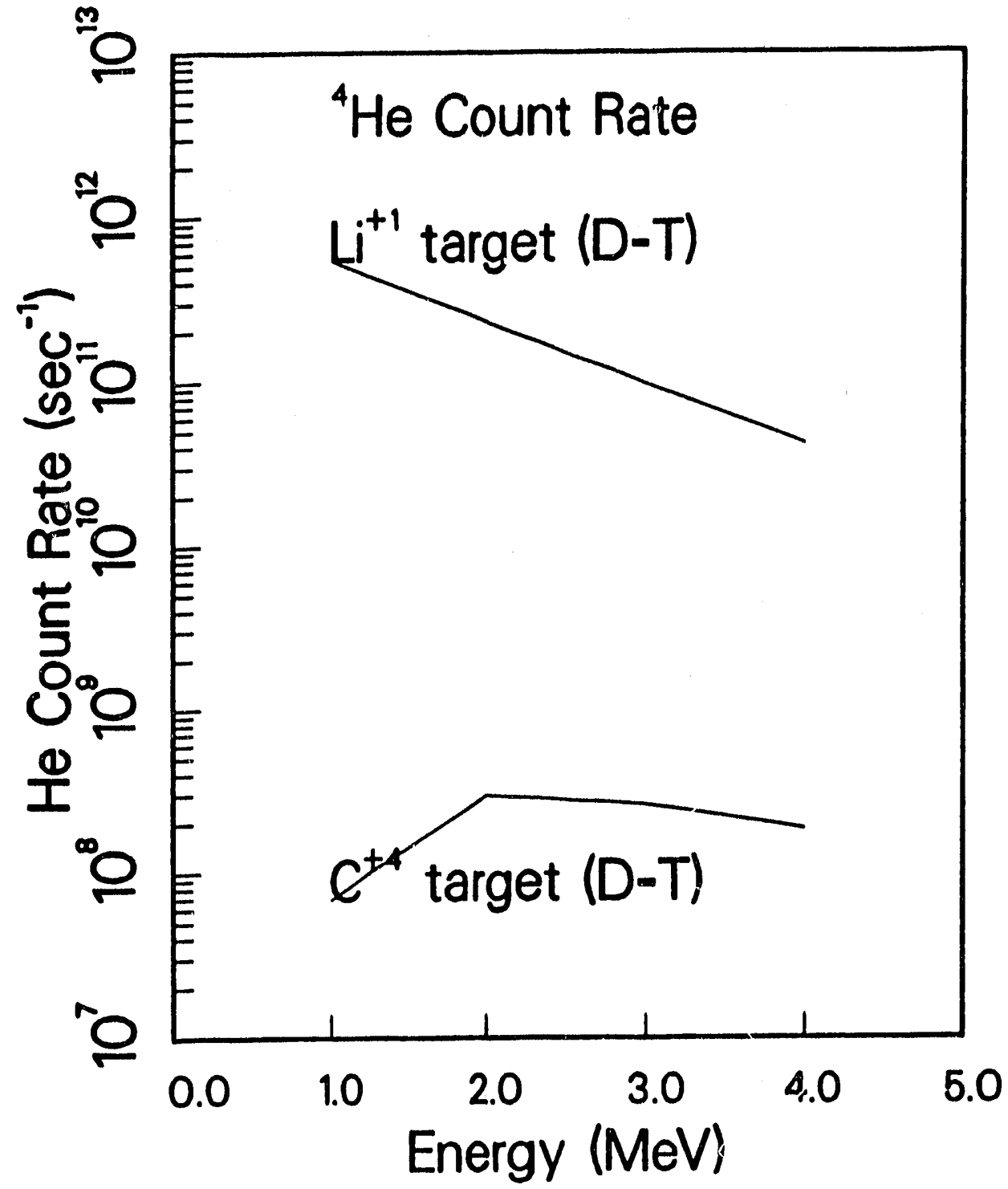

Fig. 3-7. Calculated $3.5 \mathrm{MeV}{ }^{4} \mathrm{He}$ signal during TFTR D-T experiment for Ioffe analyzer in TFTR basement. 


\subsection{HIGHLIGHTS OF FY 89 AND FY 90}

In FY89, work was concentrated on carbon pellet cloud targets. Carbon was selected due to its high heat of ablation providing good pellet penetration into the tokamak and its favorable atomic physics properties. Significant developments in FY89 included the realization that there should be a large spatial region of the ablation cloud in the helium-like state $\mathrm{C}^{4-+}$. By observing helium neutrals produced by alphas interacting with this large $\mathrm{C}^{4+}$ region, we could determine the absolute value of the fast confined alpha particle distribution.

On the experimental side, we obtained our first "snapshot" pictures of carbon pellet clouds on TEX.T. A gated intensified Xbyion CID TV camera was used to photograph line radiation from various ionization states in carbon pellet clouds. As expected, the clouds were observed to be elongated along the field lines. We observed $\mathrm{C}^{3+}$ at $5801 \AA$ but did not see any measurable $\mathrm{C}^{4+}$ radiation at either $4945 \AA$ or $8432 \AA$.

In FY90, we used a wavelength converter to attempt to observe $\mathrm{C}^{4+}$ at $2271 \AA$ from pellets injected into TEXT. We instead saw the $2297 \AA$ line of $\mathrm{C}^{2+}$. As expected, the $\mathrm{C}^{2+}$ light peaked close to the pellet while the $5801 \AA \mathrm{C}^{9+}$ light had two maximum displaced to each side of the pellet in the total magnetic field direction. We did parametric studies on cloud sizes and found the total toroidal length of the $\mathrm{C}^{3+}$ cloud along the field direction decreased strongly with increasing tokamak plasma electron density. The cloud length showed no correlation with the local tokamak electron temperature. The width of the $\mathrm{C}^{3+}$ cloud transv';se to the total magnetic field decreased slightly with the tokamak density. Paul Parks developed a model [3-2] to predict the transverse dimension of the cloud which agrees with TEXT data at $n_{\mathrm{e}}=3 \cdot 10^{13} \mathrm{~cm}^{-3}$.

In FY90, we also began a collaboration with MIT and PPPL to perform cloud measurements using the existing impurity pellet injector on TFTR. We were able to observe $\mathrm{C}^{4+}$ light at $4945 \AA$ with a total cloud length of $\sim 90 \mathrm{~cm}$ along the field. This is much larger than the $\mathrm{C}^{3+}$ clouds observed on TEXT, and is very favorable for the prospects for alpha diagnostics because the large clouds mean larger neutral signals. We were not able to do scaling studies before TFTR shut down in November 1990. 


\subsection{REFERENCES FOR SECTION 3}

[3-1] J.A. Snipes et al., Bull. Am. Phys. Soc. 36 (1991) 2377.

[3-2] P.B. Parks, Nucl. Fusion 31 (1991) 1431. 


\section{CURRENT DENSITY DIAGNOSTIC}

\subsection{FY91}

During FY91, the current density diagnostic program concentrated on preparing the $\mathrm{Li}$ beam system to operate at lower beam energy, developing an algorithm for unfolding measured beam fluorescence profiles to determine edge plasma density profiles, and developing a multichannel optical detection system compatible with the requirements for edge diagnostics.

\subsubsection{BEAM MODIFICATIONS}

For application to Texas Experimental Tokamak (TEXT) edge plasma diagnostic, the optimum energy of the neutral $\mathrm{Li}$ beam is between 15 and $30 \mathrm{keV}$, significantly lower than the $90 \mathrm{keV}$ energy previously used. Thus, near the end of FY90, the $\mathrm{Li}$ beam system was removed from TEXT (durin ${ }_{1}$, the TEXT upgrade shutdown), disassembled, and shipped back to GA. During FY91, the beamline was modified at GA to be suitable for these lower energies. Modifications to the ion optics and neutralizer cell resulted in an increase in the measured ion current to a Faraday cup located in the position of the TEXT plasma from $350 \mu \mathrm{A}$ to $2 \mathrm{~mA}$. The new ion optics results in a significant reduction in the overall beamline length, from over four met rs to about two meters. New magnetic shielding was designed and a new support table was built. At the request of the TEXT staff, that support table was put on wheels, so that the beamline could be easily removed from the port for access by other diagnostics. A schematic of the beamline vacuum system, ion optics with magnetic shielding, is shown in Fig. 4-1. The components were shipped to TEXT at the end of FY91 and the beamline was reassembled at TEXT during the first week of FY92.

\subsubsection{FLUORESCENCE DETECTION SYSTEM}

A new fluorescence detection system was designed with 20 channels viewing the beamline in the outer $10 \mathrm{~cm}$ of plasma. The specifications of the detection system came from detailed modeling of the lower energy beam performance and from the 


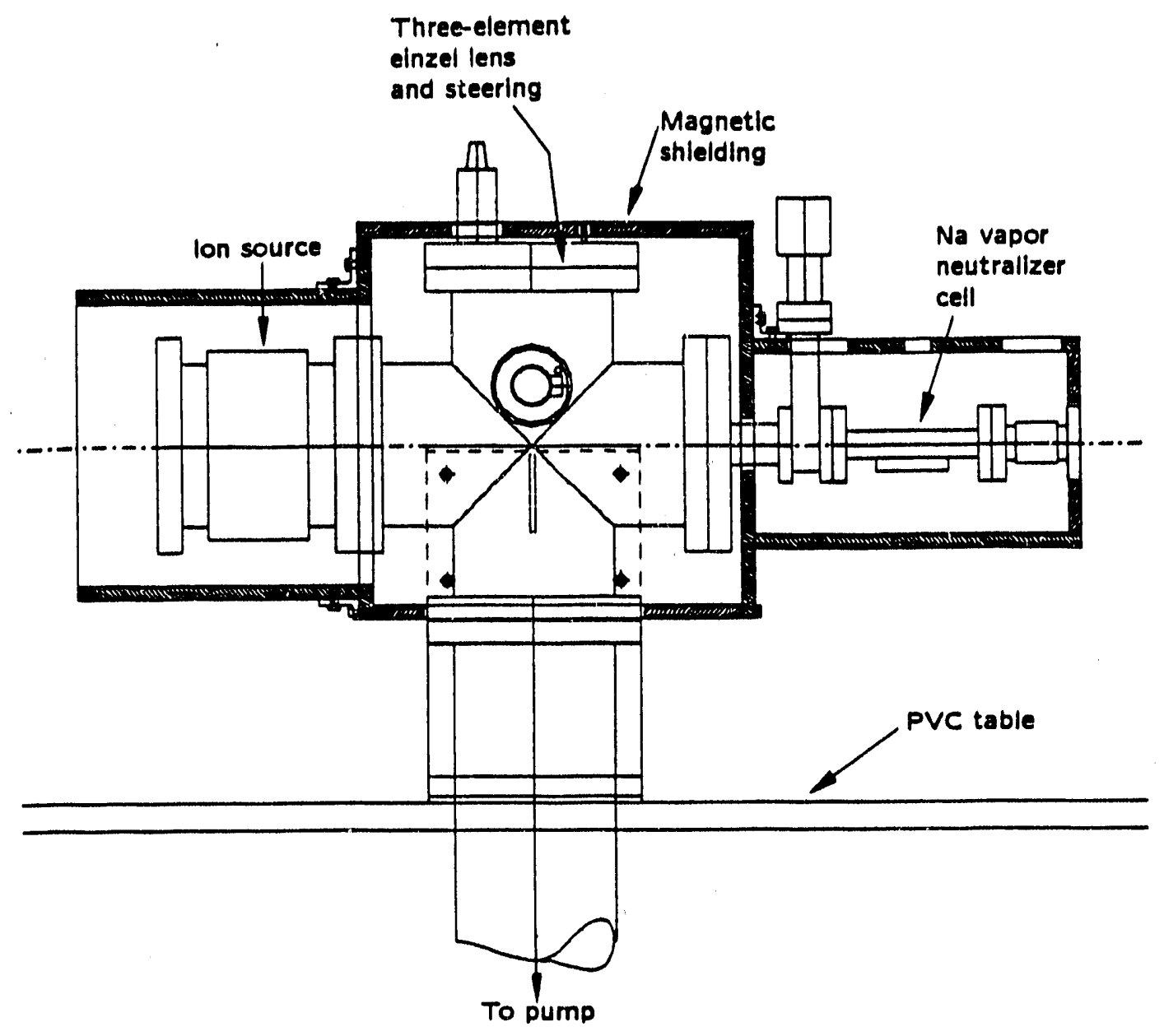

FIG. 4-1. Schematic of the $\mathrm{Li}$ beam configured for operation at energies between 15 and $30 \mathrm{keV}$.

results of the development of an unfolding algorithm discussed below. Each channel views a $6 \mathrm{~mm}$ length of beam starting abot $2 \mathrm{~cm}$ outside the limiter radius. A custom multichannel fiber bundle was designed and submitted for bids to several vendors. A bid was selected and construction of the multiple bundle system was begun. Again, the TEXT staff requested that we accommodate sharing of the port with other diagnostics, and a compatible port design was devised. Optical detectors and preamplifiers originally designed for use on the multipulse Thomson scattering system on DIII-D were chosen for use on this system. 


\subsubsection{DATA ANALYSIS}

The goal of this phase of the program is to demonstrate the ability to use the $\mathrm{Li}$ beam to measure plasma density profiles, including fluctuation phenomena, from $\mathrm{Li}$ fluorescence profiles. From our previous experience us'ng the $\mathrm{Li}$ beam, we knew of two imortant effects that influence the local beam fluorescence intensity:

1. Li beam attenuation in upstream plasma.

2. Spatial smearing due to the high velocity of the energetic $\mathrm{Li}$ atoms and the 27 nsec lifetime of the excited state.

These effects require the development of an algorithm to unfold the plasma density profiles from the measured fluorescence profiles.

Early in FY91, analysis of beam fluorescence data using a predictive $9 \mathrm{Li}$ state model showed good agreement with profiles taken with low time resolution (i.e., the effects of density fluctuations were averaged out). Thus, we are confident that our atomic physics data base is adequate for modeling the $\mathrm{Li}$ beam fluorescence. however, this predictive code is not adequate for unfolding data. Two schemes for directly unfolding the data were explored, but found to be numerically unstable. One was a direct integration of the measured profiles based on a two-state model of the $\mathrm{Li}$ atom, while the other used fast Fourier decomposition techniques. A stable and simple technique was developed, based on the fact that the beam attenuation is dominated by ion impact, while the excitation is dominated by electron impact. A simple, smooth ion density profile is then used to account for the beam attenuation, and the electron density profile, including fluctuations, can then be directly obtained from the measured profile. To test this technique, a model profile with edge fluctuations was used in the predictive nine-state code to obtain a "measured" fluorescence profile. The two profiles are show in Fig. 4-2. As can be seen, the effects of the spatial smearing are to significantly reduce the amplitude of the fluorescence fluctuations compared to the density fluctuations. Results of the inversion technique are compared to the original density profile in Fig. 4-3. The "data" points are obtained using 20 regularlyspaced points along the predicted fluorescence profile and adding in random noise. A reasonably accurate picture of the original density profile is obtained. In practice, the required smooth ion density profile can be obtained from the multichord FIR interferometer system on I'EXT. The results of this algor wa.n development have allowed us to detemine the sensitivity and minimum spatial resolution required for the detection system. 


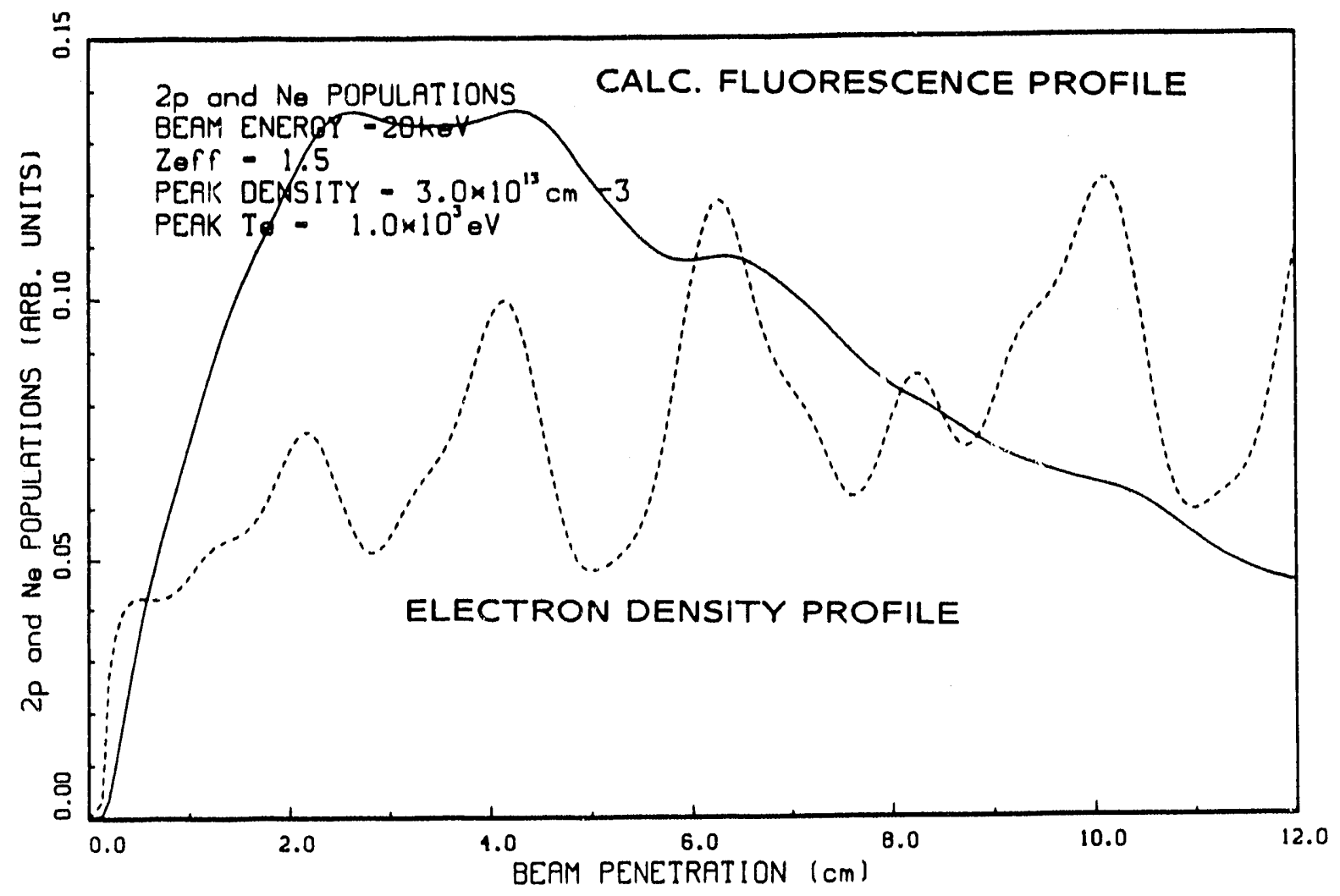

Fig. 4-2. A model edge plasma density profile including fluctuations (dashed line) and the $\mathrm{Li}$ beam fluorescence profile predicted by the nine-state code (solid line). 


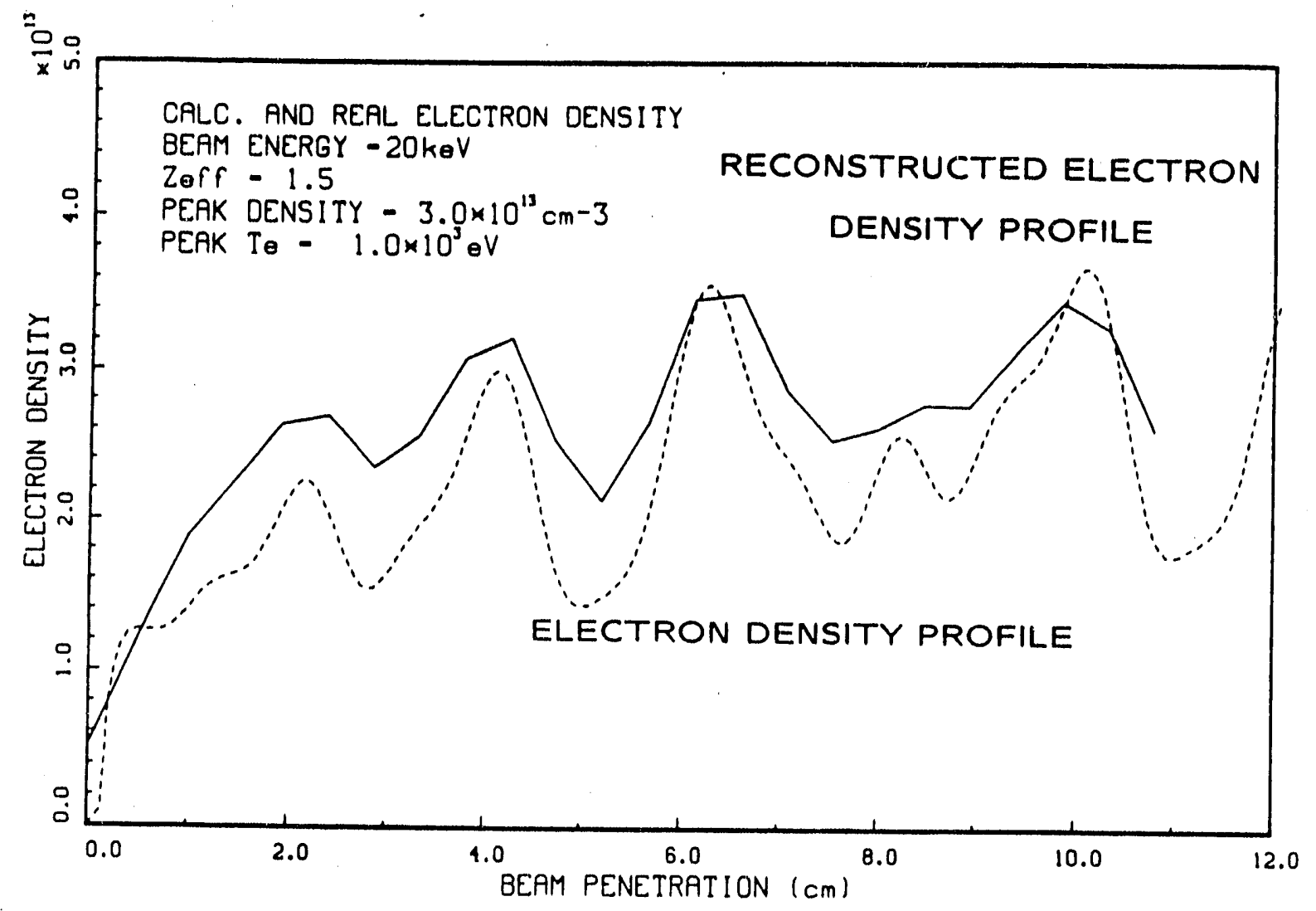

FIG. 4-3. The edge density profile derived from the fluorescence profile in Fig. 4-2 using the two-state algorithm (solid line) and the original edge plasma density profile (dashed line). 


\subsection{FY89-90 HIGHLIGHTS: CURRENT DENSITY DIAGNOSTIC}

\subsubsection{LASER ENHANCED ATTENUATION}

During FY 89 We successfully completed a proof-of-principle experiment demonstrating the Laser Enhanced Attenuation (LEA) technique for the measurement of the magnetic pitch angle on TEXT. The LEA uses resonant laser excitation of the $\mathrm{Li}$ 2S-2P transition to alter the transmission of an energetic Li beam through the plasma. Using the polarization sensitivity induced by the Zeeman effect, the local magnetic pitch angle can be measured simultaneously at several radial positions inside the plasma [4-1].

The demonstration of the viability of LEA required continued development of critical system components, as summarized in this list:

1. A transmitted $\mathrm{Li}$ beam detector for implementation on the back wall of TEXT.

2. High-bandwidth low-noise electronics for signal amplification.

3. Timing electronics for the fast-gated detection electronics.

4. A laser-wavelength monitoring system.

A Faraday cup detector with $1000 \AA$ carbon stripper foil was designed for implementation on the back wall of TEXT. This detector is reasonably durable, with a demonstrated lifetime of at least 500 discharges. The most likely candidate for failure is radiation damage to the foil from the impact of the $\mathrm{Li}$ beam itself. A schematic of the detector and high bandwidth electronics is shown in Fig. 4-4.

A successful proof-of-principle demonstration of the LEA technique using a single channel was completed. This signal was clearly identified at trigger delay times corresponding to radial positions of -4 and $+15 \mathrm{~cm}$ with approximately equal $S / N$ ratio. (Note that the previously used laser-induced fluorescence technique suffered severe drop in $S / N$ as the radius of observation decreased due to the attenuation of the $\mathrm{Li}$ beam.) The measured pitch angle as a function of time is shown in Fig. 4-5 for $r=+15 \mathrm{~cm}$, along with the total plasma current. During the times when the $S / N$ is adequate, it is clear that the measured phase is tracking the plasma current. 


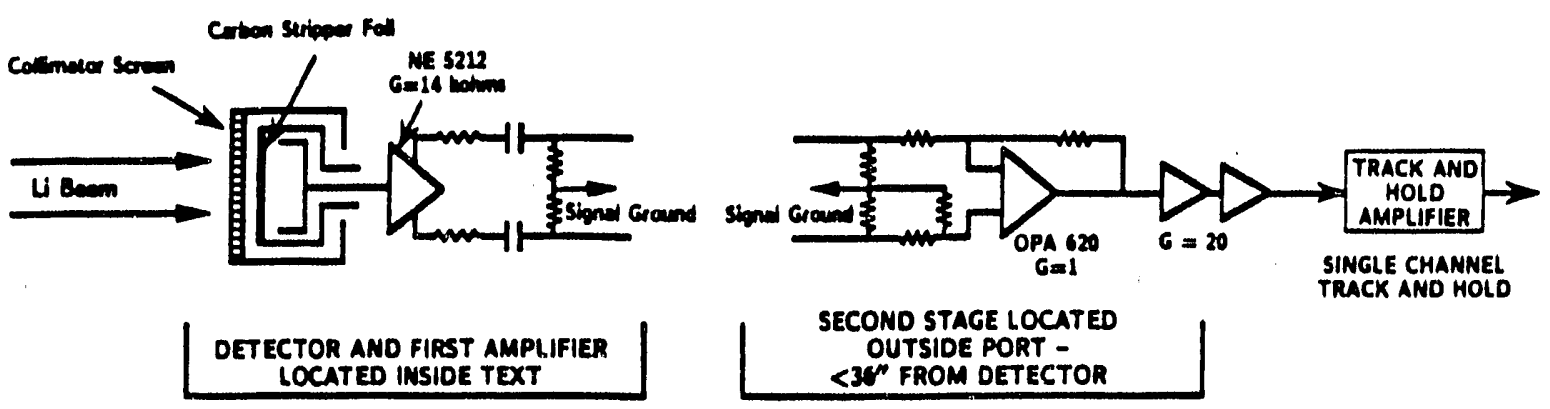

FIG. 4-4. Schematic of the transmitted beam detector with amplification and gating electronics.

\subsubsection{ZEEMAN POLARIMETRY}

We worked with the TEXT staff and L.K. Huang of Johns Hopkins to continue the development of the Zeeman polarimetry technique using the collisionally-induced fluorescence from the $\mathrm{Li}$ beam [4-2]. This work has demonstrated that adequate signal can be achieved at line average plasma densities up to about $1.5 \times 10^{13} \mathrm{~cm}^{-3}$, limited by beam attenuation. The $\mathrm{Li}$ beam provides a relatively intense, highly-localized, polarized radiation source. He has concentrated on the study of an ECH heated discharge that has shown highly peaked temperature profiles. These discharges are heated at the plasma axis, and the measured poloidal field profiles indicate that the current density is also highly peaked. Although the data analysis is preliminary, these results indicate that the $q$ on axis may be as low as 0.6 with no observable sawteeth. Presently, this is a single channel system and must be scanned between shots to obtain a radial profile.

\subsubsection{EDGE DENSITY DIAGNOSTIC USING LOW ENERGY LI BEAM}

To study the use of the $\mathrm{Li}$ beam at low beam energies $(\leqslant 30 \mathrm{keV})$ for edge plasma density studies, some modification of the $\mathrm{Li}^{+}$accelerator was required. The magnetic sector, used to bend the $\mathrm{Li}$ ion beam through a $15 \mathrm{deg}$ angle, and the accelerator column were removed from the beamline. The ion gun and electrostatic focussing optics were repositioned in order to improve the operation of the beamline at lower beam energies. Some modification to the high voltage housing, the magnetic shielding, and the vacuum plumbing was required to complete this task. After some retuning of the optics, we were able to achieve $500 \mu \mathrm{A}$ of ion current to a Faraday 

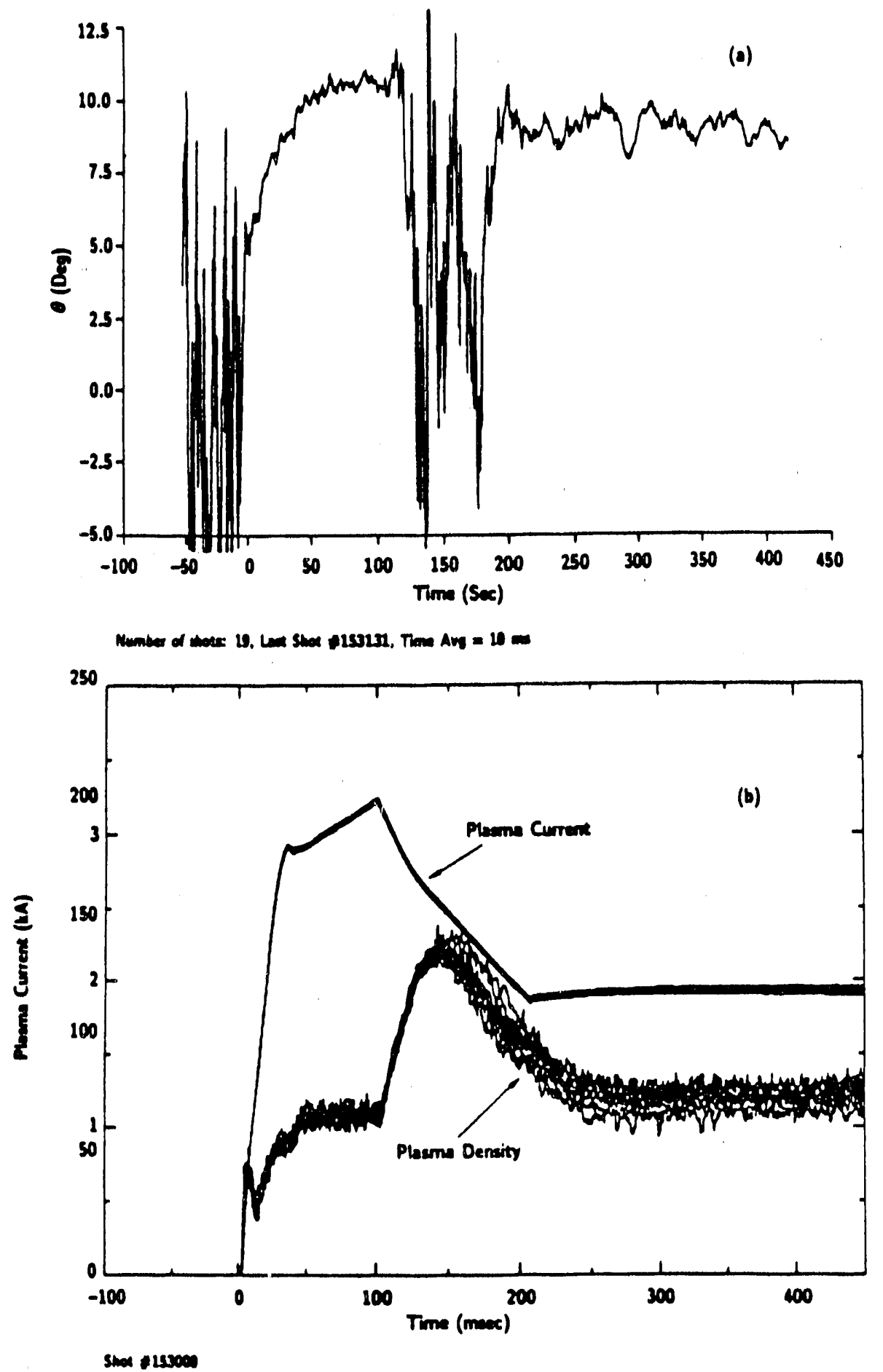

FIG. 4-5. (a) Measured pitch angle at $r=15 \mathrm{~cm}$ and (b) TEXT plasma current and density for a set of 19 discharges. 
cup (positioned after the neutralization cell) at $25 \mathrm{kV}$ beam voltage. At $15 \mathrm{kV}$, about $200 \mu \mathrm{A}$ were achieved, indicating that the current to the cup was space-charge limited. Neutralization efficiencies in the sodium vapor cell should be quite high at these energies (greater than $80 \%$ ). The total neutral current to the plasma could not be measured, but was probably somewhat lower than the Faraday cup ion current since the beam appeared to be fairly divergent.

Collisionally-induced beam fluorescence data was obtained at beam energies of 15,25 , and $30 \mathrm{keV}$ at a variety of plasma conditions. Fluorescence detectors were positioned on $1 \mathrm{~cm}$ centers at radial positions from $19 \mathrm{~cm}$ to beyond the limiter, $27 \mathrm{~cm}$. Fluorescence profiles have been compared to profiles predicted using our $\mathrm{Li}$ beam/plasma modeling code and standard "TEXT scaling" density and temperature profiles. An example is shown in Fig. 4-6. For all beam energies and all plasma conditions, the agreement is excellent. Thus, we are confident that our planning for future work using this technique on TEXT and DIII-D is accurate, and that our cross-section database is good at these beam energies.

\subsection{REFERENCES FOR SECTION 4}

[4-1] W.P. West, D.M. Thomas, and M.P. Thomas, Rev. Sci. Instrum. 59 (1988) 1617. [4-2] L.K. Huang et al., Bull. Am. Phys. Soc. 33 (1988) 2025. 


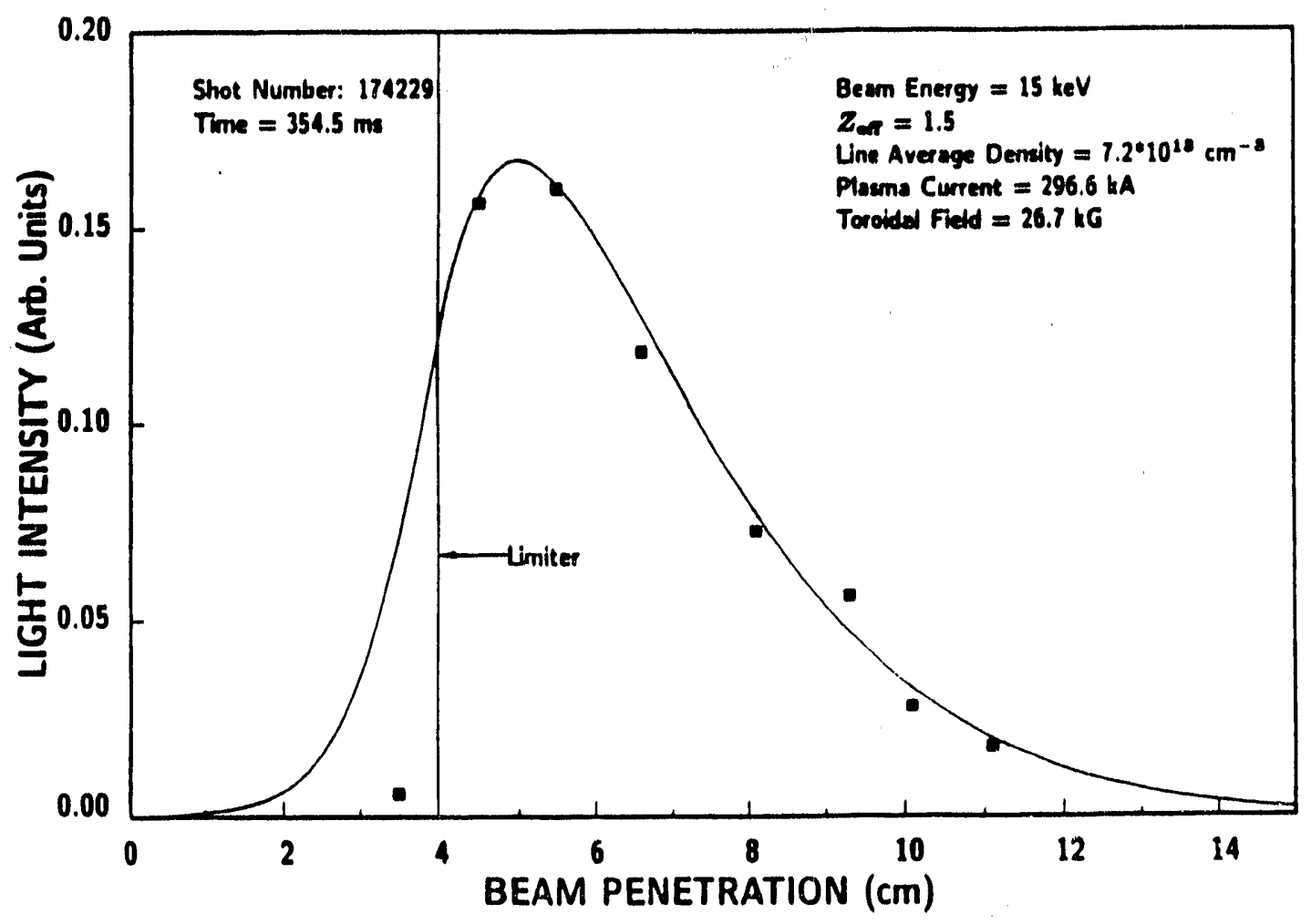

FIG. 4-6. Li beam fluorescence profiles on TEXT. The squares are measured relative fluorescence intensity as a function of distance into the plasma (the limiter is at $26 \mathrm{~cm}$ ). The solid line is predicted by the $\mathrm{Li}$ beam/plasma code (a collisional/radiative model). The code used density and temperature profiles obtained from standard "TEXT scaling." 


\section{FUSION USER SERVICE CENTER}

\subsection{OVERVIEW}

During this contract period, the User Service Center (USC) fulfilled its major goais and met all milestones. Connection to the National Energy Research Supercomputer Center (NERSC) was maintained. This was a particularly active task as NERSC phased out the satellite based MFEnet replacing it with the fiber optic/T1 besed Energy Sciences Network (ESnet). While this evolution was planned and coordinated by the NERSC staff, significant participation was required by the USC as various

equipment was removed, installed, and/or upgraded and new software was installed and tested.

Local computational facilities were provided for the GA Fusion community. Based on capacity planning, runtime statistics, and budgetary guidance, the VAX cpu cycles were upgraded and UNIX was introduced. The Local Area Network was expanded so that ethernet was available as needed in offices for workstations, XTerminals, Appletalk, and printers. Support was provided for various user needs, from UNIX training classes to developing a security plan to accommodate the computational needs of visitors from sensitive countries.

The USC archived a substantial amount of DIII-D data as the shot size rose in excess of 50 Mbytes. Online disk storage was increased by 3 Gbytes. The VAX Cluster storage controller was upgraded allowing for more throughput. Helical scan $8 \mathrm{~mm}$ tape storage was implemented in order to condense the offline data storage. Software for data retrieval was modified and optimized for maximum performance.

\subsection{FY91 MAJOR ACCOMPLISHMENTS}

The ESnet became fully functional. The MFE satellite ground station, including the related computer equipment, was deinstalled and removed. All hardware pertaining to the MFEnet was returned to NERSC. There were several microcode upgrades to the ESnet cisco router during the year. The ESnet performed exceptionally well, with noticeable improvements in throughput over the MFEnet. 
The Multiflow computer was not highly utilized. Although some physics calculations were done, most users shied away from the UNIX operating system. The EFITD code and associated data translation programs were converted. A problem with the DISSPLA software from Computer Associates (CA) which made the software unusable was never fully resolved by CA. Without DISSPLA, the output from EFTID could not be interpreted. Due to lack of user interest and poor vendor support the decision was made to decommission the Multiflow. The Multiflow minisupercomputer was deinstalled and returned to GE Capital in September after an early buyout of the lease was arranged.

The USC developed an upgrade plan to increase the VAX computer power after extended measurements showed the VAXes were very loaded during prime time causing poor response. The upgrade plan was presented to management, the user community, and sent out to other Fusion labs for peer review. The accepted plan replaced two obsolete VAXes with two VAX 4000-300s, moved one 8650 to DIII-D, and added a VAX workstation and terminal servers. There was a total increase in VAX MIPS at the USC of 16 and 4.5 at DIII-D. The Implementation Plan describing the USC VAX upgrade was approved by the DOE/SAN office. The equipment was procured from DEC and installed in August. The VAX 4000-300s are general purpose computers, accessible to all Fusion users, while the VAXstation is dedicated to EFITD calculations during run periods. Before these VAXes could be joined to the existing USC cluster, all systems were upgraded to VMS 5.4-2. Access by terminal to these VAXes also required the upgrading of the Xyplex communication equipment to the LAT protocol. A second S1032 database license was purchased for one of the VAX 4000-300s. CIQBAs from Micro Technologies, Inc. were procured and installed on both VAX 4000-300s. These boards made the VAX 4000-300s full cluster members rather than satellite nodes with the end result being a dramatic improvement in system performance.

Multinet version 3.0 was installed on all USC VAXes. Domain Name Service was configured and implemented replacing the static routing tables and thus ensuring that the most current routing addresses are always available. NFS was activated making the VAX disks available to all the workstations. Three Fastcomm 9600 baud modems were installed on the USC Micom, enabling users to access the Fusion computers directly at 9600 baud. The Appletalk network in the Theory area was completed. Any computer on the entire Fusion ethernet can be directly accessed from any Macintosh in Theory. All LECnet restrictions at the USC have been removed allowing worldwide 
access to the computers through DECnet. The restrictions are still in force for the DIII-D computers. NCD XTerminals were received and installed. The ethernet concentrator was also upgraded and expanded to accommodate the XTerminals and the additional workstations.

The printer evaluation begun last year was completed with the purchase of a LPS20 Printstation from DEC. It is the only generally accessible Postscript printer on the USC network.

The Computer Security Plan for sensitive visitors was modified. This modification expanded the access on the USC VAXes for the visitors from sensitive countries, and thereby improved the computational collaboration with other DIII-D participants. This modified plan was approved by the DOE/SAN. The USC Statement of Strategy was also updated and submitted to DOE/SAN. It, too, was subsequently approved. Login banners on all Fusion computers were modified to reflect the DOE directives. The USC participated in a CIAO security workshop held at Lawrence Berkeley Laboratory.

The switch at NERSC from the CTSS operating system to UNICOS created a great deal of work for GA. Many of the production physics codes being run on the Crays were written using the CIVIC fortran compiler. These need to be converted to CFT77 fortran and compiled under UNICOS. The USC assigned one FTE to this project. The first major GA code to be converted was the ONETWO Transport Code.

The NCAR graphics package was purchased for all USC platforms. MATLAB was procured and installed on the Theory workstation. The visiting physicists from DFRC in Cadarache, France demonstrated the usefulness of this program for if modeling using the ALCYON code on their Cray. Having MATLAB locally will allow GA Fusion to make effective use of the ALCYON code.

Optimization of EFIT has been undertaken by the USC. The first step involved two major modifications to the IMSL bicubic spline routines in an effort to improve performance. To date, about a $15 \%$ overall reduction in cpu time has been realized.

A survey of high end RISC workstations was undertaken. Arrangements were made to run the EFITD benchmark on DEC, IBM, Sun, Silicon Graphics, Cray, and HP platforms. The ultimate goal is to procure a standard high end workstation and allow for parallel code development, thus positioning the DIII-D program to take 
advantage of the most cost effective computer cycles available. Procurement and implementation is planned for early FY92.

The USC was transferred from the Administration Group to the Fusion Group reporting to the Fusion Computer Systems. This realignment of the USC into the main stream of the Fusion Group made for a more efficient organization. The USC staff offices were also relocated. The USC computers remain in their present location, the GA main computer room. One of the two vacant positions in the USC was filled. 

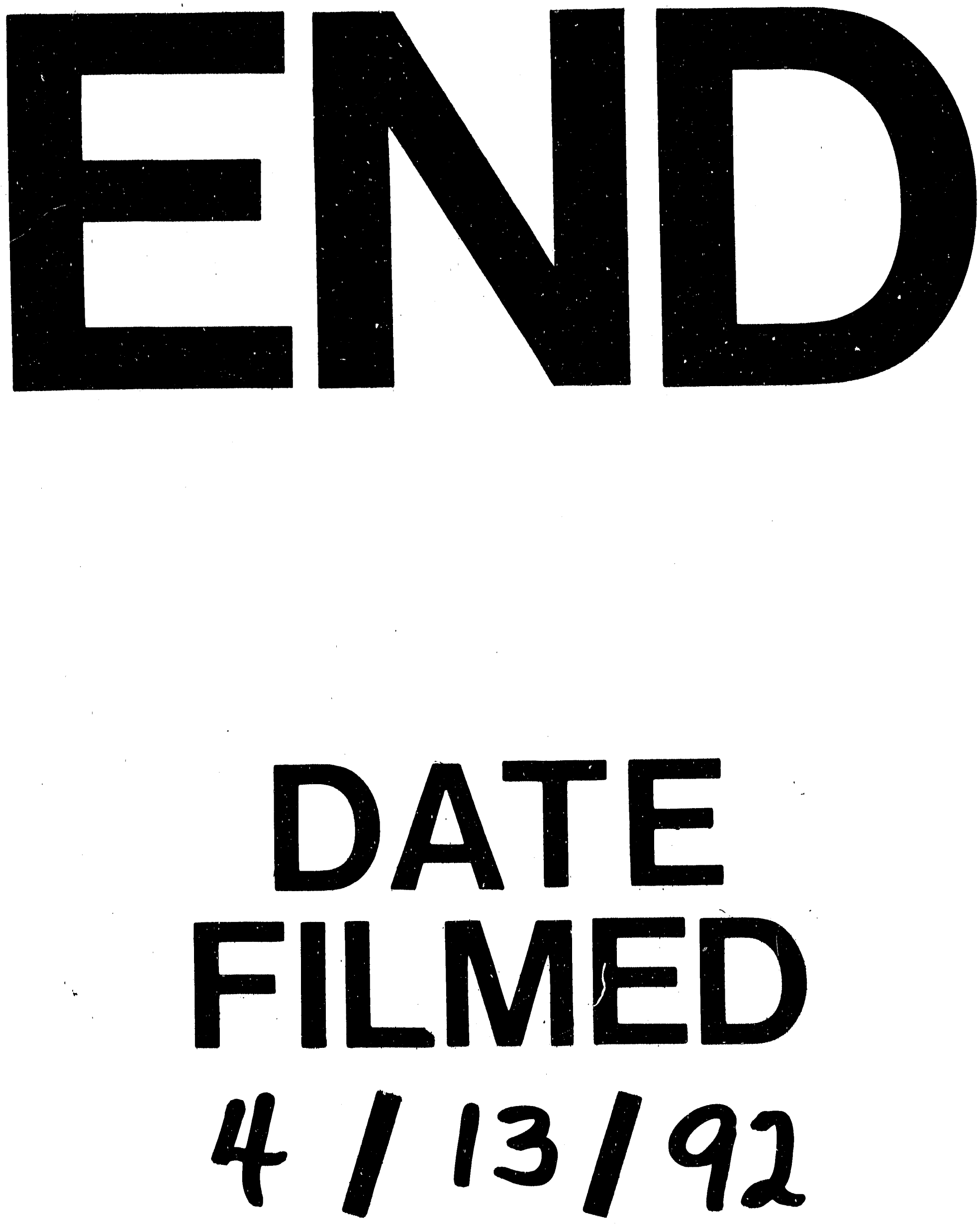


$$
\text { ב. }
$$

